\title{
Fluctuation Modes of a Twist-Bend Nematic Liquid Crystal
}

\author{
Z. Parsouzi, ${ }^{1}$ S. M. Shamid, ${ }^{1}$ V. Borshch, ${ }^{2}$ P. K. Challa, ${ }^{1}$ A. R. Baldwin, ${ }^{1}$ M. G. Tamba, ${ }^{3}$ C. Welch, ${ }^{3}$ G. H. Mehl, ${ }^{3}$ \\ J. T. Gleeson, ${ }^{1}$ A. Jakli, ${ }^{2}$ O. D. Lavrentovich, ${ }^{2}$ D. W. Allender, ${ }^{1}$ J. V. Selinger, ${ }^{2}$ and S. Sprunt, ${ }^{1,}$ \\ ${ }^{1}$ Department of Physics, Kent State University, Kent, Ohio 44242, USA \\ ${ }^{2}$ Chemical Physics Interdisciplinary Program and Liquid Crystal Institute, Kent State University, \\ Kent, Ohio 44242, USA \\ ${ }^{3}$ Department of Chemistry, University of Hull, Hull HU6 7RX, United Kingdom \\ (Received 30 November 2015; revised manuscript received 29 February 2016; published 22 June 2016)
}

\begin{abstract}
We report a dynamic light-scattering study of the fluctuation modes in a thermotropic liquid crystalline mixture of monomer and dimer compounds that exhibits the twist-bend nematic $\left(\mathrm{N}_{\mathrm{TB}}\right)$ phase. The results reveal a spectrum of overdamped fluctuations that includes two nonhydrodynamic modes and one hydrodynamic mode in the $\mathrm{N}_{\mathrm{TB}}$ phase, and a single nonhydrodynamic mode plus two hydrodynamic modes (the usual nematic optic axis or director fluctuations) in the higher temperature, uniaxial nematic phase. The properties of these fluctuations and the conditions for their observation are comprehensively explained by a Landau-de Gennes expansion of the free-energy density in terms of heliconical director and helical polarization fields that characterize the $\mathrm{N}_{\mathrm{TB}}$ structure, with the latter serving as the primary order parameter. A "coarse-graining" approximation simplifies the theoretical analysis and enables us to demonstrate quantitative agreement between the calculated and experimentally determined temperature dependence of the mode relaxation rates.
\end{abstract}

DOI: 10.1103/PhysRevX.6.021041

Subject Areas: Condensed Matter Physics, Soft Matter

\section{INTRODUCTION}

The twist-bend nematic $\left(\mathrm{N}_{\mathrm{TB}}\right)$ phase is a fascinating new addition to the family of orientationally ordered, liquid crystalline states of matter. It has been described as the "fifth nematic phase" [1], complementing the uniaxial, biaxial, chiral helical (cholesteric), and blue phase nematics. Originally proposed by Meyer [2], and later elaborated on theoretically by Dozov [3], the existence of the $\mathrm{N}_{\mathrm{TB}}$ phase was suggested experimentally [4] and subsequently confirmed [1,5] in low-molecular-weight liquid crystals (LCs) containing achiral dimers having an oddnumbered hydrocarbon linkage between the mesogenic ends. Interest in these materials was also inspired by simulation studies [6], which predicted a nematic-nematic transition in LC dimers with odd-numbered linkages.

The $\mathrm{N}_{\mathrm{TB}}$ state possesses some remarkable properties. First, the average local molecular long axis (specified by a unit vector $\hat{\mathbf{n}}$ called the director) simultaneously bends and twists in space. In the case of LC dimers with odd linkage, the specific tendency to bend is presumably caused by an all-trans conformation of the molecules, which results in a pronounced bent shape. The addition of twist allows the bend to be uniform everywhere in space. The combination

\footnotetext{
*ssprunt@kent.edu
}

Published by the American Physical Society under the terms of the Creative Commons Attribution 3.0 License. Further distribution of this work must maintain attribution to the author(s) and the published article's title, journal citation, and DOI. of bend and twist produces an oblique helicoidal (or heliconical) winding of the director (Fig. 1), with a temperature-dependent cone angle $\beta$ (angle between $\hat{\mathbf{n}}$ and the helicoidal axis) of magnitude ranging up to approximately $30^{\circ}$ [5,7]. This differs from an ordinary cholesteric LC phase, where a pure twist of $\hat{\mathbf{n}}$ results in a right-angle helicoid $\left(\beta=90^{\circ}\right)$.

Second, the helicoidal pitch in the $\mathrm{N}_{\mathrm{TB}}$ phase is on a molecular scale-i.e., on the order of $10 \mathrm{~nm}[1,5]-$ compared with cholesterics, where the supramolecular pitch typically exceeds $100 \mathrm{~nm}$. The much larger pitch of a cholesteric may be attributed to the relative freedom of rotations around the long molecular axes, when the latter are orthogonal to the helical axis $\left(\beta=90^{\circ}\right)$. This configuration mitigates the chiral part of intermolecular interactions [8]. By contrast, in the $\mathrm{N}_{\mathrm{TB}}$ state (with $\beta<90^{\circ}$ ), the bend-imposed hindrance of molecular rotations results in a much shorter, nanoscale modulation, which, however, remains purely orientational in nature-i.e., there is no associated variation in mass density (no Bragg peak detected by x-ray scattering $[1,4,5])$.

Third, and again unlike a cholesteric, the component molecules of $\mathrm{N}_{\mathrm{TB}}$-forming LCs are typically achiral. Thus, the chiral nature of the helicoidal structure is spontaneously generated, with degenerate domains of left- and righthanded helicity.

Finally, although the $\mathrm{N}_{\mathrm{TB}}$ phase shows no evidence of a macroscopic polarization, the flexoelectric effect [9] associated with spontaneous bending of $\hat{\mathbf{n}}$ and the recent observation of an electroclinic effect [10] in the $\mathrm{N}_{\mathrm{TB}}$ phase 


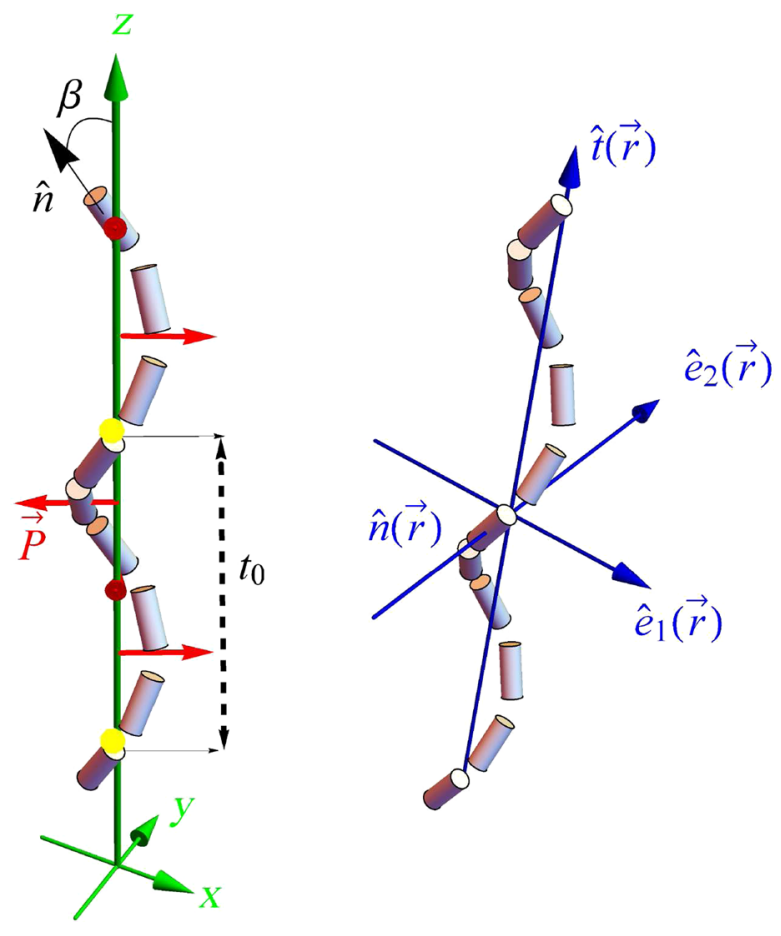

FIG. 1. Left diagram: Schematic representation of the $\mathrm{N}_{\mathrm{TB}}$ phase structure, showing heliconical director $\hat{\mathbf{n}}$ (with cone angle $\beta$ and helical pitch $t_{0}$ ) and helical polarization field $\mathbf{P}$. Right diagram: Frame of reference used to describe spatial variations of the average director or pitch axis, $\hat{\mathbf{t}}$, on length scales much longer than the pitch (see Sec. IV). The orthogonal unit vectors $\hat{\mathbf{e}}_{1}$ and $\hat{\mathbf{e}}_{2}$ form a right-handed system with $\hat{\mathbf{t}}$. The $x y z$ axes are fixed in the laboratory frame.

suggest that a short-pitch helical polarization field is tied to the heliconical director structure (see Fig. 1). A recent theory [11] describing the transition between uniaxial and twist-bend nematic phases invokes such a polarization field as the primary order parameter.

Despite the intense experimental and theoretical efforts to explore the $\mathrm{N}_{\mathrm{TB}}$ phase, the nature of collective fluctuation modes associated with the short-pitch helicoidal structure remains an open question. It is a vital one to address since the spectrum and dispersion of these modes are closely related to the basic structural features and to the relevant order parameter(s) and because properties of the fluctuations provide an important test of theories describing the formation of the $\mathrm{N}_{\mathrm{TB}}$ state. Although previous dynamic light scattering (DLS) measurements [12] revealed a softening of the elastic constant associated with bend distortions of the director above the $\mathrm{N}-\mathrm{N}_{\mathrm{TB}}$ transition, they did not probe fluctuation modes specifically associated with the heliconical $\mathrm{N}_{\mathrm{TB}}$ structure. Here, we report, to the best of our knowledge, the first DLS study of fluctuations within the $\mathrm{N}_{\mathrm{TB}}$ phase and their critical behavior near the transition. Our measurements reveal a pair of strongly temperature-dependent nonhydrodynamic modes plus a single hydrodynamic mode in the $\mathrm{N}_{\mathrm{TB}}$ phase, and a single nonhydrodynamic mode and pair of hydrodynamic modes (the usual director modes of a uniaxial nematic) in the higher-temperature nematic phase. We demonstrate excellent agreement between the behavior of the observed modes and new theoretical predictions based on a "coarsegrained" version of a Landau-de Gennes free energy for the nematic-to- $\mathrm{N}_{\mathrm{TB}}$ transition [11].

The coarse-graining approximation, inspired by significant earlier theoretical work on cholesterics $[13,14]$ and appropriate in the limit of helical pitch much shorter than an optical wavelength, treats surfaces of constant phase in the heliconical structure as "pseudolayers." Within this approximation, which has previously been used to explain the effect of high magnetic fields on the $\mathrm{N}_{\mathrm{TB}}$ phase [15] and to account for its flow properties [16], the normal fluctuation modes involving the director may be mapped onto those of a chiral smectic-A phase, with effective layer spacing equal to the pitch, effective director parallel to the local pitch axis, and effective elastic constants that arise from the short-pitch orientational modulation rather than from a true mass density wave.

An alternative approach to coarse-graining the $\mathrm{N}_{\mathrm{TB}}$ phase has recently been proposed by Meyer and Dozov [17]. It is based on a negative bend elastic constant model of an unstable nematic that induces the twist-bend state [3], and it describes the coarse-grained $\mathrm{N}_{\mathrm{TB}}$ phase as an effective chiral smectic-A phase, with smecticlike elastic constants. Expressions for these are given in terms of parameters $\beta$ and $q_{0}$ of the $\mathrm{N}_{\mathrm{TB}}$ phase and the Frank nematic elastic constants. The model predicts one hydrodynamic and one nonhydrodynamic fluctuation mode in the $\mathrm{N}_{\mathrm{TB}}$ state, analogous to the layer compression or bending and layer tilt modes of an ordinary smectic, and it accounts for some (but not all) of our experimental results.

While our coarse-graining approach is generally consistent with that of Ref. [17], it provides significant new elements that (1) enable a complete explanation of the modes observed in our experiment (including an additional nonhydrodynamic mode observed in both $\mathrm{N}_{\mathrm{TB}}$ and nematic phases) and (2) relate all of the modes to microscopic fluctuations of the polarization as well as the director field. Our theoretical approach highlights the central role of a helical polarization field in describing the nematic-to- $\mathrm{N}_{\mathrm{TB}}$ transition - an aspect that fundamentally distinguishes the $\mathrm{N}_{\mathrm{TB}}$ phase from the other known nematic LC states, including, in particular, the cholesteric phase.

It is worthwhile to briefly review other theoretical studies that describe the twist-bend nematic state and its transition to the uniform uniaxial nematic. Lelidis and Barbero [18] further develop the elastic theory of Ref. [3] by explicitly examining the Euler-Lagrange equation that minimizes a free-energy functional that depends only on first derivatives of the director out to quartic order. Kats and Lebedev [19] developed a Landau theory based on a two-component vector order parameter $\boldsymbol{\phi}($ where $\boldsymbol{\phi} \cdot \hat{\mathbf{n}}=0)$, varying on the 
$10 \mathrm{~nm}$ scale along the direction of the nematic director $\hat{\mathbf{n}}$, as the twist-bend phase order parameter. Alternatively, Virga [20] and Barbero et al. [21] introduce a helix axis $\hat{\mathbf{t}}$, with $\hat{\mathbf{n}} \cdot \hat{\mathbf{t}}=\cos \beta$, and analyze the elastic properties of $\hat{\mathbf{t}}$. Reference [21] further analyzes the uniform nematic to a twist-bend nematic transition. Also, Greco et al. [22] have extended the concepts of the molecular-level mean-field Maier-Saupe model for rodlike molecules to the case of bent-core dimeric molecules. The onset of the twist-bend nematic is characterized by a polar-order parameter along with a pitch and cone angle. The polar order parameter has some features in common with the polarization in the flexoelectric model of Ref. [11], but a detailed comparison has not been made in order to elucidate differences.

The nature of the fluctuation modes based on each of these theories has not been published as far as we know, except for some discussion in Ref. [19] of a hydrodynamic mode in the twist-bend phase corresponding to pseudolayer rippling.

The body of this paper is organized as follows: In Sec. II, we provide essential details about the experimental setup and procedures, while Sec. III describes the key experimental results. Section IV presents a detailed discussion of a Landau theory for a $\mathrm{N}-\mathrm{N}_{\mathrm{TB}}$ transition and the coarsegraining approach to calculate the normal fluctuation modes associated with the twist-bend structure. The theoretical predictions are compared to the experimental results in Sec. V, and Sec. VI summarizes our findings and offers some concluding remarks.

\section{EXPERIMENTAL DETAILS}

DLS measurements were performed on a 30/70 wt $\%$ mixture of the monomer and dimer compounds shown in Fig. 2 [23]. This mixture has the phase sequence isotropic $\rightarrow$ (uniaxial) nematic $(\mathrm{N}) \rightarrow \mathrm{N}_{\mathrm{TB}} \rightarrow$ crystal in cooling, with a N-to- $\mathrm{N}_{\mathrm{TB}}$ transition temperature, $T_{\mathrm{TB}}=94.2^{\circ} \mathrm{C}$ (measured with a calibrated platinum resistance thermometer in our light-scattering oven). The $\mathrm{N}_{\mathrm{TB}}$ phase in this system has been characterized by a variety of techniques [5]; for our purposes, its choice afforded the possibility to obtain high-quality alignment of the average director (optic axis) in either homogeneous planar or homeotropic configurations-i.e., with average $\hat{\mathbf{n}}$ parallel or normal to the plane of the optical substrates, respectively — using thin $(5 \mu \mathrm{m})$ cells with appropriate surface treatments.

Our DLS measurements utilized two depolarized scattering geometries-G1 and G2, depicted in Fig. 2-in which homodyne time correlation functions of the depolarized scattered intensity of laser light (wavelength $\lambda=532 \mathrm{~nm}$ ) are collected as a function of scattering vector q and temperature $T$.

In geometry G1 (Fig. 2), the average director is planar aligned and oriented perpendicular to the scattering plane. We set the wave vector $\mathbf{k}_{i}$ of the incident beam to an angle $\theta_{i}=0^{\circ}$ (measured with respect to the substrate normal) and

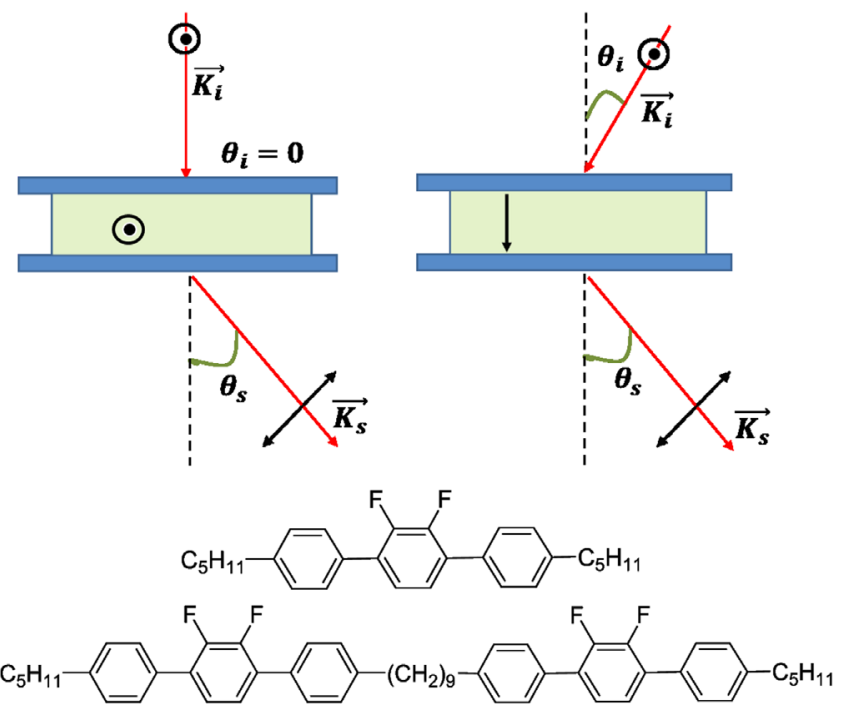

FIG. 2. Top diagram: Light-scattering geometries G1 (left) and G2 (right) described in the text, with the average director (optic axis) in the sample cell indicated by the arrow pointing out of the page for G1 (homogeneous planar alignment with average $\hat{\mathbf{n}}$ normal to the scattering plane) or the downward arrow for G2 (homeotropic alignment with average $\hat{\mathbf{n}}$ in the plane). The orientations of polarizer and analyzer are similarly indicated. Bottom diagram: Chemical structure of the monomer and dimer compounds utilized for the present study. The 30/70 wt $\%$ mixture exhibits a $\mathrm{N}-\mathrm{N}_{\mathrm{TB}}$ phase transition at $94.2{ }^{\circ} \mathrm{C}$.

varied the direction of wave vector $\mathbf{k}_{s}$ of the scattered light (described by scattering angle $\theta_{s}$ relative to the substrate normal). In the nematic phase, for large $\theta_{s}$, this geometry probes nearly pure splay fluctuations of the director with relaxation rate $\Gamma_{1}^{n} \sim q^{2}$.

In geometry $\mathrm{G} 2$, the average director is parallel to the substrate normal (homeotropic alignment) and lies in the scattering plane; in this case, depolarized DLS in the nematic phase probes a combination of overdamped twist and bend fluctuations of $\hat{\mathbf{n}}$-the hydrodynamic twist-bend director mode, with relaxation rate $\Gamma_{2}^{n} \sim q^{2}$. The incident wave vector $\mathbf{k}_{i}$ was fixed at $\theta_{i}=15^{\circ}$ or $35^{\circ}$, while the direction of $\mathbf{k}_{s}$ was varied between $\theta_{s}=-10^{\circ}$ and $50^{\circ}$, with respect to average $\hat{\mathbf{n}}$. When $\theta_{s}=0^{\circ}, \mathbf{k}_{s}$ lies along $\langle\hat{\mathbf{n}}\rangle$, and the scattering from director fluctuations is nominally extinguished ("dark director" geometry). This choice of $\theta_{s}$ provides an opportunity to detect fluctuation modes that do not originate from $\hat{\mathbf{n}}$ and contribute to the dielectric tensor in their own right.

\section{RESULTS}

Figure 3 shows polarizing microscope images of a homeotropic sample of the mixture during the uniaxial nematic-to-twist-bend $\left(\mathrm{N}-\mathrm{N}_{\mathrm{TB}}\right)$ transition, with the lower left part of each picture corresponding to the $\mathrm{N}$ and the upper right part to the $\mathrm{N}_{\mathrm{TB}}$ phase. Figure 3(a) confirms the high quality of the homeotropic alignment of 


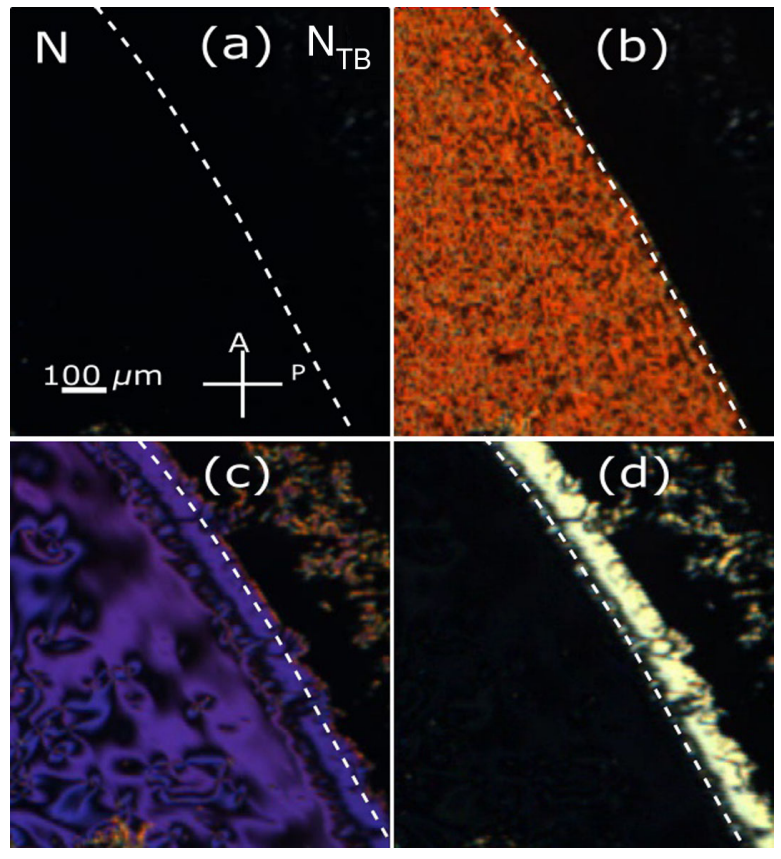

FIG. 3. Polarizing microscope textures for a 5- $\mu \mathrm{m}$-thick homeotropically aligned sample of the studied mixture. The optic axis is normal to the image plane, and the sample is placed between crossed polarizers. (a) Separate regions of $\mathrm{N}$ and $\mathrm{N}_{\mathrm{TB}}$ phases observed at the transition between the two; the boundary is marked by the dashed line. Both regions are uniform and dark, indicating high-quality homeotropic alignment of the director $\hat{\mathbf{n}}$ in the nematic and pitch axis $\hat{\mathbf{t}}$ in the $\mathrm{N}_{\mathrm{TB}}$ phase. (b) Under an applied ac voltage (5 V @10 KHz), a second-order Freedericsz transition (reorientation of $\hat{\mathbf{n}}$ in the center of the sample) is observed in the nematic region, while the $\mathrm{N}_{\mathrm{TB}}$ region is unchanged. (c) Under higher voltage (7 V @ $10 \mathrm{KHz})$, the $\mathrm{N}_{\mathrm{TB}}$ region undergoes a first-order reorientation of $\hat{\mathbf{t}}$ in the form of nucleating toroidal focal conic domains (FCDs) and expanding stripes of splay and saddle-splay deformations of $\hat{\mathbf{t}}$. (d) Several seconds after the voltage has been switched off, the nematic region relaxes back to the homeotropic state, whereas the $\mathrm{N}_{\mathrm{TB}}$ region relaxes considerably slower.

the average director and its persistence across the $\mathrm{N}-\mathrm{N}_{\mathrm{TB}}$ transition. In the $\mathrm{N}_{\mathrm{TB}}$ phase, the average value of $\hat{\mathbf{n}}$ is the pitch axis $\hat{\mathbf{t}}$ of the heliconical structure, which is oriented perpendicular to the substrates (image plane in the figure). Under an applied ac voltage (5 V @ $10 \mathrm{KHz}$ ), a secondorder Freedericsz transition (reorientation of the average director in the center of the sample) is observed in the $\mathrm{N}$ region, while the $\mathrm{N}_{\mathrm{TB}}$ region is unchanged [Fig. 3(b)]. In the $\mathrm{N}_{\mathrm{TB}}$ region, the reorientation occurs at a higher voltage [7 V @ $10 \mathrm{KHz}$, Fig. 3(c)], and in the form of propagating focal-conic domains (FCDs), such as is usually observed in smectic liquid crystals with negative dielectric anisotropy [24,25]. The "pseudolayered" nature of the heliconical structure [15] is reflected in the gradual relaxation of the FCDs to homeotropic alignment after removal of the field. As Fig. 3(d) indicates, the slow relaxation rate and presence
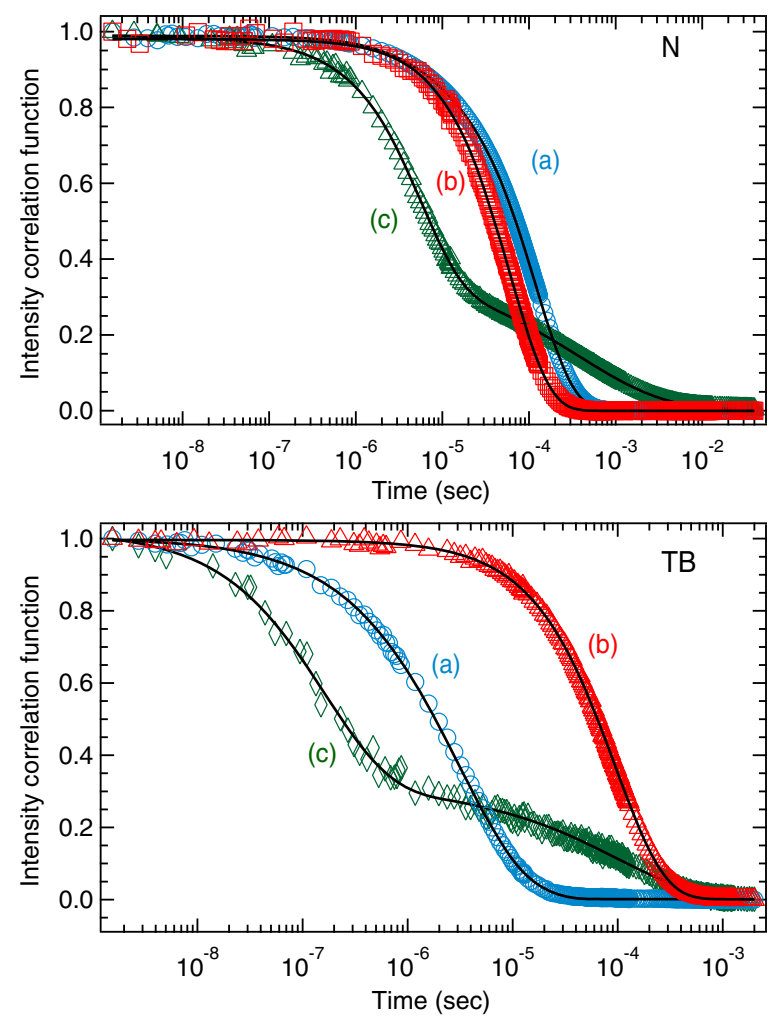

FIG. 4. Top panel: Normalized homodyne DLS correlation functions taken in the nematic phase of the studied LC mixture for (a) geometry $\mathrm{G} 2$ with $T-T_{\mathrm{TB}}=16.2^{\circ} \mathrm{C}$ and angles $\theta_{i}=15^{\circ}, \theta_{s}=40^{\circ}$, (b) $\mathrm{G} 1$ with $T-T_{\mathrm{TB}}=6.0^{\circ} \mathrm{C}$ and $\theta_{i}=0^{\circ}$, $\theta_{s}=60^{\circ}$, and (c) $\mathrm{G} 2$ with $T-T_{\mathrm{TB}}=16.2^{\circ} \mathrm{C}$ and $\theta_{i}=15^{\circ}, \theta_{s}=$ $0^{\circ}$ ("dark" director geometry). Solid lines represent fits to a single exponential decay, except for (c), which is fit to a double exponential with the slower component stretched. Bottom panel: Normalized correlation data taken in the $\mathrm{N}_{\text {Тв }}$ phase for (a) geometry G2 with $T-T_{\mathrm{TB}}=-0.93^{\circ} \mathrm{C}$ and $\theta_{i}=15^{\circ}, \theta_{s}=40^{\circ}$, (b) G1 with $T-T_{\mathrm{TB}}=-1.1^{\circ} \mathrm{C}$ and $\theta_{i}=0^{\circ}, \theta_{s}=60^{\circ}$, and (c) G2 with $T-T_{\mathrm{TB}}=-2.5^{\circ} \mathrm{C}$ and $\theta_{i}=35^{\circ}, \theta_{s}=0^{\circ}$ (dark director geometry). Solid lines are single exponential fits, except for a double exponential in (c).

of FCDs are quite distinct from the behavior observed in the nematic phase.

Figure 4 displays representative normalized DLS correlation functions recorded in the nematic and twist-bend phases of $5-\mu \mathrm{m}$-thick samples of the LC mixture for geometries G1 and G2. In the "splay" geometry (G1), a single overdamped fluctuation mode is detected in both $\mathrm{N}$ and $\mathrm{N}_{\mathrm{TB}}$ phases. By scanning $\theta_{s}$, we determined $\Gamma_{1}^{n} \sim q^{2}$ with $\Gamma_{1}^{n} / q^{2}$ in the range $10^{-11}-10^{-10} \mathrm{~s}^{-1} \mathrm{~m}^{2}$. Thus, splay fluctuations of the optic axis are hydrodynamic on both sides of the transition.

The spectrum and behavior of modes detected in geometry G2 are more interesting. In the nematic phase (above $T_{\mathrm{TB}}$ ), two overdamped modes are observed in the range of $\theta_{s}$ studied: the expected hydrodynamic twist-bend director mode with relaxation rate $\Gamma_{2}^{n} \sim q^{2}$ (see measured 


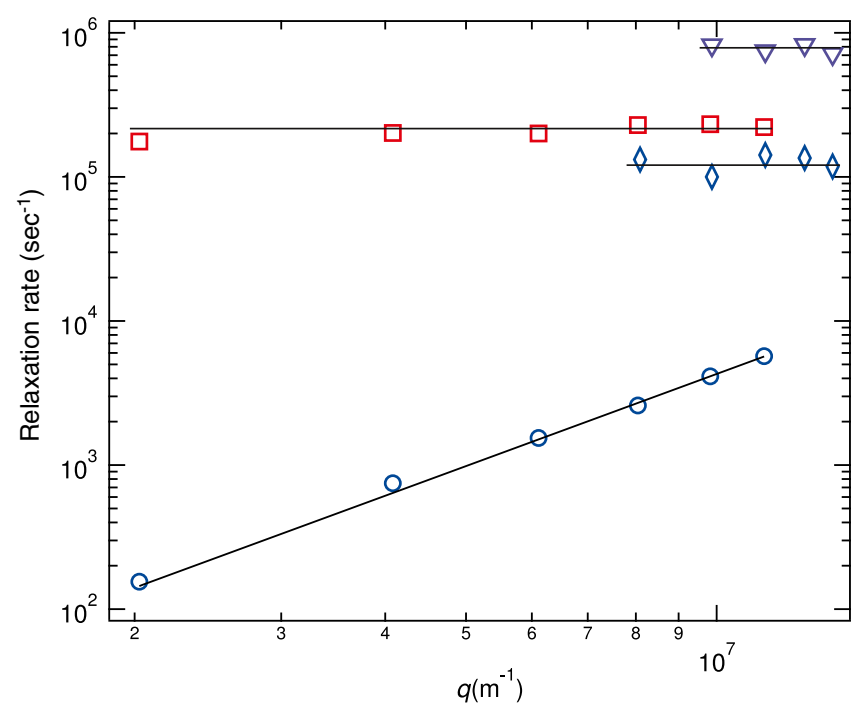

FIG. 5. Dependence of the relaxation rates of the fluctuation modes detected in geometry G2 on the magnitude of the scattering vector $q$. Circles and squares correspond to relaxation rates $\Gamma_{2}^{n}$ and $\Gamma_{2}^{p}$ of the hydrodynamic director and nonhydrodynamic polarization modes detected in scattering geometry G2 in the middle of the nematic phase $\left(T-T_{\mathrm{TB}}=25^{\circ} \mathrm{C}\right)$. The scattering angles $\theta_{s}$ range from $-5^{\circ}$ to $45^{\circ}$ for fixed incident angle $\theta_{i}=15^{\circ}$ (see definitions in Fig. 2, top right). The slope of the line through the data on the log-log plot for $\Gamma_{2}^{n}$ is 2 , indicating $\Gamma_{2}^{n} \sim q^{2}$. Diamonds and triangles correspond to relaxation rate $\Gamma_{2}^{t}$ of the nonhydrodynamic pitch axis fluctuations at temperatures $T-T_{\mathrm{TB}}=-0.85^{\circ} \mathrm{C}$ and $-8.0^{\circ} \mathrm{C}$, respectively, in the $\mathrm{N}_{\mathrm{TB}}$ phase. These data are limited to higher $q$ (or $\theta_{s}$ in the range $25^{\circ}$ or $35^{\circ}$ to $65^{\circ}$ ) due to a large component of background scattering at lower $q$, whose effect is exacerbated because of the low scattering intensity from fluctuations in the $\mathrm{N}_{\mathrm{TB}}$ phase in the $\mathrm{G} 2$ geometry.

$q^{2}$ dependence in Fig. 5) of order $\sim 10^{3} \mathrm{~s}^{-1}$ and $\Gamma_{2}^{n} / q^{2} \simeq 10^{-11}-10^{-10} \mathrm{~s}^{-1} \mathrm{~m}^{2}$, and a faster, nonhydrodynamic mode (Fig. 5) with $\Gamma_{2}^{p} \simeq 10^{5} \mathrm{~s}^{-1}$ and independent of $q$. (The meaning of superscript $p$ will be clarified in the next section.) The relaxation rates of both modes were extracted from fits of the correlation data to double exponential decays.

The presence of the fast mode in the DLS correlation function is most evident in the "dark director" geometry where $\theta_{s}=0^{\circ}$ [see data labeled (c) in the top panel of Fig. 4], although it contributes weakly for $\theta_{s} \neq 0^{\circ}$. However, even in the "dark" geometry where fluctuations in $\hat{\mathbf{n}}$ do not contribute to the DLS to first order, we still observe the decay of the slow director mode with a significant spread in its relaxation rate. (The fit in this case used a stretched exponential, with one additional fitting parameter.) Alignment mosaicity and a consequent broadening of the scattered wave vector $\mathbf{k}_{s}$ relative to $\hat{\mathbf{n}}$ could produce a "leakage" of the slow director mode, but that does not account for the fact that no significant spread in $\Gamma_{2}^{n} \sim q^{2}$ is observed for $\theta_{s}$ off the "dark" condition. An alternative scenario based on an intrinsic coupling between the fast and slow fluctuations is argued in Sec. V.

In the $\mathrm{N}_{\mathrm{TB}}$ phase, the relaxation rates and $q$ dependence of the modes observed in geometry G2 change significantly. The twist-bend director mode, which dominates the scattering for $\theta_{s} \neq 0$, develops a large energy gap; its relaxation rate increases markedly below the transition $\left(T=T_{\mathrm{TB}}\right)$ to values in the $10^{5}-10^{6} \mathrm{~s}^{-1}$ range, and, as evidenced in Fig. 5, becomes $q$ independent. Thus, below $T_{\mathrm{TB}}$, the twist-bend mode crosses over from a hydrodynamic to nonhydrodynamic mode. As we demonstrate in Sec. V, the magnitude of the gap is consistent with a modulation of $\hat{\mathbf{n}}$, whose period agrees with results from freeze-fracture transmission-electron-microscopy (FFTEM) [5] for the nanoscale periodic structure of the $\mathrm{N}_{\mathrm{TB}}$ phase. Since the effective director (or optic axis) is the pitch axis $\hat{\mathbf{t}}$, for clarity we label its relaxation rate as $\Gamma_{2}^{t}$ (replacing $\Gamma_{2}^{n}$ ).

Correlation data taken in the "dark director" geometry (G2 with $\theta_{s}=0^{\circ}$ ) in the $\mathrm{N}_{\mathrm{TB}}$ phase reveal a second, even faster nonhydrodynamic mode with a relaxation rate of $10^{6}-10^{7} \mathrm{~s}^{-1}$ [see data labeled (c) in the bottom panel of Fig. 4], about 10 times higher than the values of $\Gamma_{2}^{p}$ for the fast mode in the nematic phase detected in the same geometry. Additionally, and again as in the nematic, a slow process - with relaxation rate comparable to that of a hydrodynamic director mode-also contributes to the correlation function.

In both phases, the total scattering intensity in the dark geometry, $\theta_{s}=0^{\circ}$, is about 10 times weaker than the intensity for neighboring angles $\theta_{s}= \pm 10^{\circ}$, where the twist-bend director mode couples to the dielectric tensor and dominates the scattering.

Figure 6 shows the temperature dependence of the relaxation rates for the two nonhydrodynamic modes $\left(\Gamma_{2}^{t}\right.$ and $\Gamma_{2}^{p}$ ) in the $\mathrm{N}_{\mathrm{TB}}$ phase, and for the nonhydrodynamic mode $\left(\Gamma_{2}^{p}\right)$ and hydrodynamic director mode $\left(\Gamma_{2}^{n}\right)$ in the nematic phase (see figure inset). These results were obtained from analysis of correlation data taken at fixed $\theta_{i}, \theta_{s}$ in geometry G2. The nonhydrodynamic modes clearly slow down significantly on their approach to $T_{\mathrm{TB}}$ from both sides of the transition, although on the lowtemperature side, the present data are limited to temperatures further than $0.9^{\circ} \mathrm{C}$ from the transition.

The data points in the $\mathrm{N}_{\mathrm{TB}}$ phase are less dense, especially close to the transition. The reason is twofold: First, the scattered intensity from the fluctuation modes is generally much lower in the $\mathrm{N}_{\mathrm{TB}}$, so obtaining high-quality homodyne correlation functions with good statistics was not always possible during a temperature scan, especially given variations in background scattering that we speculate arise from evolving boundaries between left- and righthanded chiral domains. Second, data for the faster nonhydrodynamic mode were recorded in the dark director geometry, where the scattered intensity was even lower and 

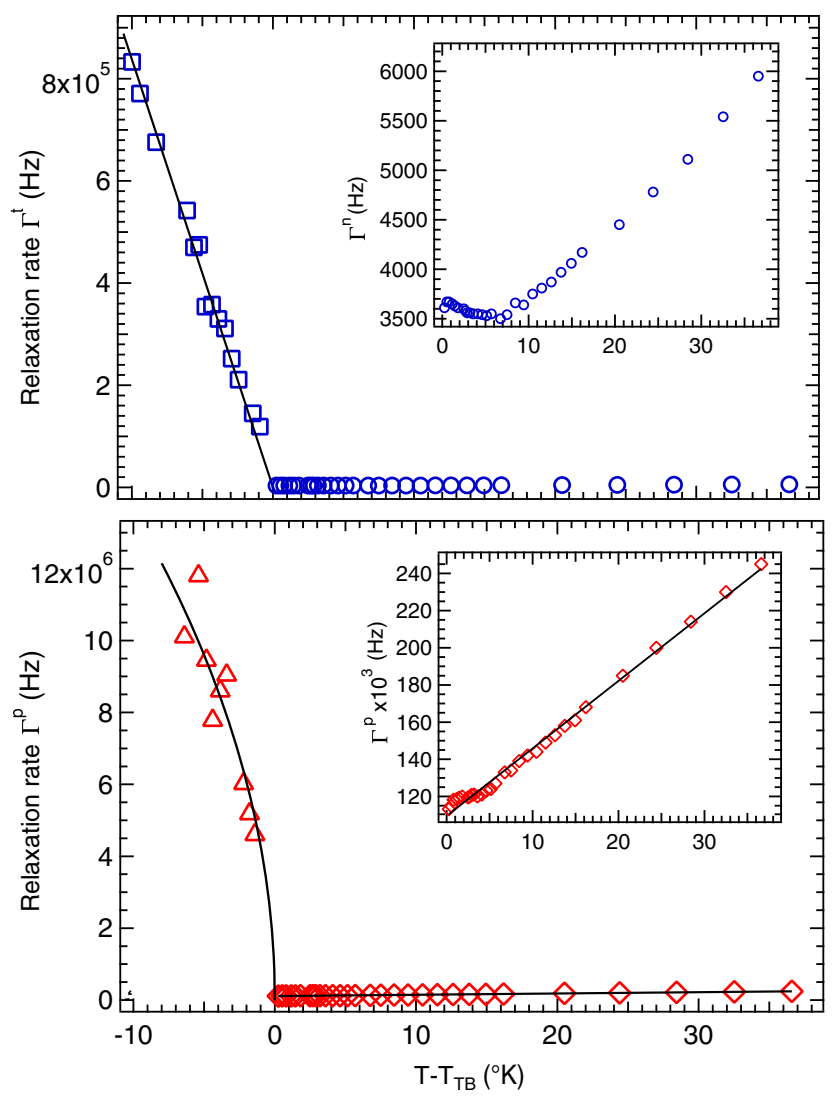

FIG. 6. Top panel: Temperature dependence of relaxation rates associated with director fluctuations detected in scattering geometry G2 in the uniaxial nematic phase ( $\Gamma^{n}$ for $T>T_{\mathrm{TB}}$, circles in main figure and inset) and twist-bend phase ( $\Gamma^{t}$ for $T<T_{\mathrm{TB}}$, squares in main figure) with fixed $\theta_{i}=15^{\circ}$ and $\theta_{s}=40^{\circ}$ (see Fig. 2, top right). Bottom panel: Temperature dependence of the relaxation rate of polarization fluctuations in the nematic (diamonds in main figure and inset for $\theta_{s}=40^{\circ}$ and $\theta_{i}=15^{\circ}$ ) and $\mathrm{N}_{\mathrm{TB}}$ (triangles in main figure for $\theta_{s}=0^{\circ}, \theta_{i}=35^{\circ}$ ) phases. The solid lines in both panels are fits of $\Gamma^{t}$ and $\Gamma^{p}$ to calculated results from the coarse-grained free-energy density model presented in Sec. IV of the text.

the accurate homodyne correlation function even more difficult to obtain.

Finally, the temperature dependence of the inverse total scattered intensity $\left(I_{2}^{-1}\right)$, recorded in geometry G2, is plotted in Fig. 7. These data were taken at fixed $\theta_{i}=15^{\circ}, \theta_{s}=40^{\circ}$, where the dominant signal in the $\mathrm{N}_{\mathrm{TB}}$ phase comes from the nonhydrodynamic mode corresponding to $\Gamma_{2}^{t}$, and in the nematic phase from the hydrodynamic twist-bend director mode corresponding to $\Gamma_{2}^{n}$. As $T \rightarrow T_{\mathrm{TB}}$ from below, the decrease in $I_{2}^{-1}$ mirrors the decrease in $\Gamma_{2}^{t}$ (Fig. 6).

\section{THEORY}

A successful model for the experimental fluctuation spectrum must account for (1) the crossover from two hydrodynamic modes and one nonhydrodynamic mode in

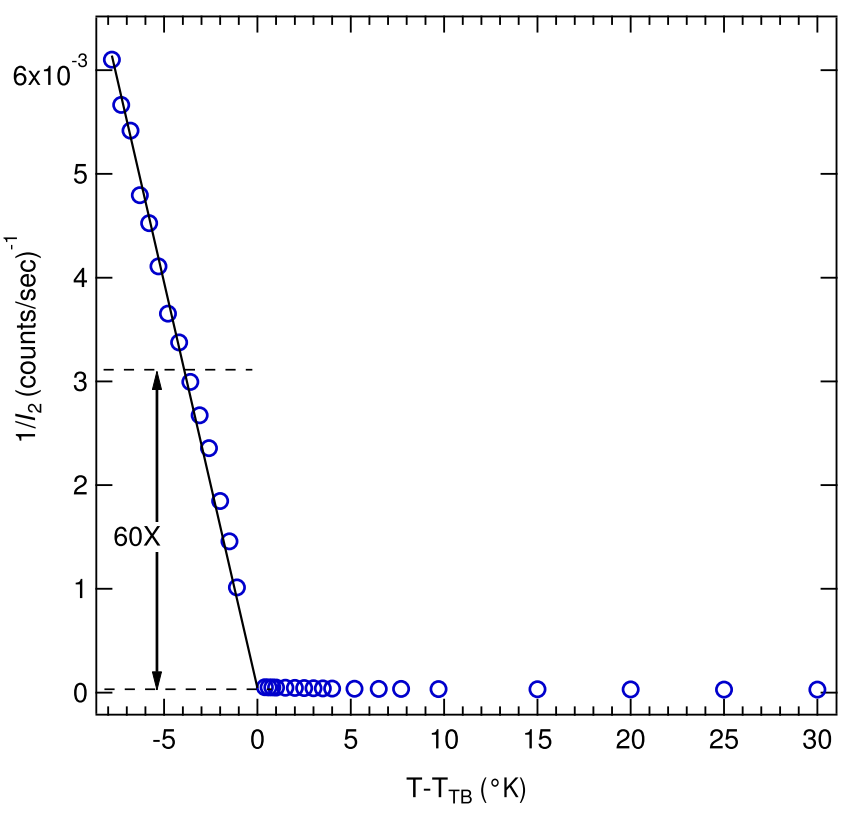

FIG. 7. Temperature dependence of the inverse of the total scattering intensity $I_{2}^{-1}$ recorded in geometry G2 for $\theta_{i}=15^{\circ}$, $\theta_{s}=40^{\circ}$. The solid line is a linear fit of the data for $T<T_{\mathrm{TB}}$.

the nematic to one hydrodynamic mode and two nonhydrodynamic modes in the $\mathrm{N}_{\mathrm{TB}}$ phase, (2) the identity of the faster (nonhydrodynamic) mode detected in each phase, (3) the coupling of this fast process to slower director modes (evidenced in the data from the dark director geometry), and (4) the temperature dependence of the relaxation rates of the nonhydrodynamic modes. To this end, we require a model free-energy density for the nematic to twist-bend transition that contains relevant hydrodynamic and nonhydrodynamic fields, and the appropriate coupling between them.

Without developing a detailed theory, one could simply deduce the basic nature and number of modes in the $\mathrm{N}_{\mathrm{TB}}$ phase by exploiting the analogy between the $\mathrm{N}_{\mathrm{TB}}$ and chiral smectic-A phase mentioned in the Introduction. This analogy, which is useful when the length scale probed experimentally is much greater than the heliconical pitch, predicts the following: one hydrodynamic mode that combines pseudolayer bending and compression (with effective elastic constants $K_{\text {eff }}$ and $B_{\text {eff }}$, respectively), one nonhydrodynamic mode that involves tilt of the average director relative to the pseudolayer normal (and an additional elastic constant $D_{\text {eff }}$ ), and assuming chirality produces a polarization, an additional nonhydrodynamic mode due to fluctuations in a polarization field (parallel to the pseudolayers), which is coupled to director tilt.

As noted in Sec. III, we have indeed observed one hydrodynamic and two nonhydrodynamic modes in the $\mathrm{N}_{\mathrm{TB}}$ phase, although the main focus of our present experiments is the nonhydrodynamic modes. However, the above-mentioned analogy by itself does not tell us how 
the elastic constants $K_{\text {eff }}, B_{\text {eff }}, D_{\text {eff }}$ depend on temperature, or how they are composed of more microscopic parameters that characterize the $\mathrm{N}_{\mathrm{TB}}$ structure. Nor does the analogy explain the nonhydrodynamic mode observed in the highertemperature nematic phase.

We therefore analyze the fluctuation modes starting from a Landau-de Gennes expansion of the free-energy density in terms of the heliconical $\mathrm{N}_{\mathrm{TB}}$ director and a helical polarization field, and then subsequently (via a coarsegraining approximation) obtain expressions for $K_{\text {eff }}, B_{\text {eff }}$, $D_{\text {eff }}$. While alternative Landau-de Gennes models, such as those presented in Refs. [17] and [19], may also account for the spectrum of hydrodynamic and nonhydrodynamic modes we detect in the $\mathrm{N}_{\mathrm{TB}}$ phase, our model, including polarization fluctuations, successfully describes the nature of these modes on both sides of the transition and, in particular, their temperature dependence on the $\mathrm{N}_{\mathrm{TB}}$ side.

Let $\mathbf{P}$ represent a polarization field (vector order parameter) that originates, e.g., from the transverse dipole moment associated with the bent conformation of the dimer molecules, which promotes the formation of the $\mathrm{N}_{\text {TВ }}$ phase. It is convenient to use a dimensionless form for this order parameter, $\mathbf{p}=\mathbf{P} / P_{\text {sat }}$, where $P_{\text {sat }}$ corresponds to the saturated polarization at low temperatures.

The free-energy density expanded in terms of the fields $\hat{\mathbf{n}}$ and $\mathbf{p}$ reads [11]

$$
\begin{aligned}
F_{\mathrm{NTB}}= & \frac{K_{1}}{2}(\nabla \cdot \hat{\mathbf{n}})^{2}+\frac{K_{2}}{2}(\hat{\mathbf{n}} \cdot \nabla \times \hat{\mathbf{n}})^{2} \\
& +\frac{K_{3}}{2}[\hat{\mathbf{n}} \times(\nabla \times \hat{\mathbf{n}})]^{2}+\frac{\mu}{2}|\mathbf{p}|^{2}+\frac{\nu}{4}|\mathbf{p}|^{4} \\
& +\frac{\kappa}{2}(\nabla \mathbf{p})^{2}-\Lambda[\hat{\mathbf{n}} \times(\nabla \times \hat{\mathbf{n}})] \cdot \mathbf{p}+\eta(\hat{\mathbf{n}} \cdot \mathbf{p})^{2} .
\end{aligned}
$$

Here, $K_{1}, K_{2}$, and $K_{3}$ are the Frank elastic constants for splay, twist, and bend distortions of the director $\hat{\mathbf{n}}$. The coefficient $\mu=\mu_{0}\left(T-T_{0}\right)$ is the temperature-dependent Landau coefficient for the polarization $\mathbf{p}\left(\mu_{0}\right.$ being a constant), while $\nu>0$ is a higher-order, temperatureindependent Landau coefficient. The elastic constant $\kappa$ penalizes spatial distortions in $\mathbf{p}$, and the coefficient $\Lambda$ couples $\mathbf{p}$ with bend distortions. The last term (not included in Ref. [11]), with $\eta>0$, favors polarization perpendicular to the nematic director and is consistent with bend flexoelectricity. Because $\mathbf{p}$ is defined to be dimensionless, the Landau coefficients $\mu$ and $\nu$ carry the same units, and $\kappa$ has the same units as the Frank constants.

In the $\mathrm{N}_{\mathrm{TB}}$ phase, the director field has the heliconical modulation

$$
\hat{\mathbf{n}}=\hat{\mathbf{z}} \cos \beta+\hat{\mathbf{x}} \sin \beta \cos \left(q_{0} z\right)+\hat{\mathbf{y}} \sin \beta \sin \left(q_{0} z\right),
$$

with pitch wave number $q_{0}$ and cone angle $\beta$. (Note that $\sin \beta$ was called $a$ in Ref. [11].) We assume the polarization field has the helical modulation

$$
\mathbf{p}=\hat{\mathbf{x}} p_{0} \sin \left(q_{0} z\right)-\hat{\mathbf{y}} p_{0} \cos \left(q_{0} z\right)
$$

with magnitude $p_{0}$, perpendicular to $\hat{\mathbf{n}}$ and to the pitch axis $\hat{\mathbf{z}}$ [26], as shown in Fig. 1 (left side). In the nematic phase, $\beta$ and $p_{0}$ are both zero, while $q_{0}$ is undefined; in the $\mathrm{N}_{\mathrm{TB}}$ phase, these quantities all become nonzero.

We proceed by inserting Eqs. (2) and (3) into Eq. (1) for $F_{\mathrm{NTB}}$, and then minimizing with respect to $q_{0}, \beta$, and $p_{0}$ in order to obtain relations among these parameters in the ground state. For this calculation, we follow Ref. [11] and generalize it to the case of the weak polar elastic constant $\kappa$, which will turn out to be physically relevant. First, minimization with respect to $q_{0}$ gives

$$
q_{0}=\frac{\Lambda p_{0} \sin \beta \cos \beta}{\kappa p_{0}^{2}+K_{3} \sin ^{2} \beta \cos ^{2} \beta+K_{2} \sin ^{4} \beta},
$$

and minimization with respect to $\beta$ gives

$$
\sin ^{2} \beta=-\frac{\kappa p_{0}^{2}}{K_{2}}+\sqrt{\frac{\kappa p_{0}^{2}}{K_{2}}\left(1+\frac{\kappa p_{0}^{2}}{K_{2}}\right)},
$$

or

$$
\sqrt{\frac{\kappa}{K_{2}}} p_{0}=\frac{\sin ^{2} \beta}{\sqrt{\cos 2 \beta}} .
$$

Substituting Eqs. (4) and (5) into the free-energy density and expanding for small $p_{0}$ and $\kappa$ gives

$$
\begin{aligned}
F_{\mathrm{NTB}}= & \frac{1}{2}\left[\mu_{0}\left(T-T_{0}\right)-\frac{\Lambda^{2}}{K_{3}}\right] p_{0}^{2} \\
& +\frac{\Lambda^{2} \kappa^{1 / 2} K_{2}^{1 / 2}}{K_{3}^{2}}\left|p_{0}\right|^{3}+\frac{1}{4} \nu p_{0}^{4} .
\end{aligned}
$$

From this form of the effective free-energy density, we can see that there is a second-order transition from the nematic to the $\mathrm{N}_{\mathrm{TB}}$ phase at the temperature

$$
T_{\mathrm{TB}}=T_{0}+\frac{\Lambda^{2}}{K_{3} \mu_{0}} .
$$

This transition is unusual because the relative magnitudes of the cubic and quartic terms in Eq. (6) depend on the relative smallness of $p_{0}$ and $\kappa$. Close to the transition, where $p_{0} \ll\left(4 \Lambda^{2} \kappa^{1 / 2} K_{2}^{1 / 2}\right) /\left(K_{3}^{2} \nu\right)$, the cubic term dominates over the quartic term. By minimizing the effective free energy, we see that $p_{0}$ depends on temperature as

$$
p_{0}(T)=\frac{K_{3}^{2} \mu_{0}\left(T_{\mathrm{TB}}-T\right)}{3 \Lambda^{2} \kappa^{1 / 2} K_{2}^{1 / 2}} .
$$

This result is consistent with the scaling reported in Ref. [11], with a slight correction in the numerical 
coefficient. By contrast, farther from the transition, where $p_{0} \gg\left(\Lambda^{2} \kappa^{1 / 2} K_{2}^{1 / 2}\right) /\left(K_{3}^{2} \nu\right)$, the quartic term dominates over the cubic term, and the prediction for $p_{0}$ becomes

$$
p_{0}(T)=\sqrt{\frac{\mu_{0}\left(T_{\mathrm{TB}}-T\right)}{\nu}} .
$$

From the general form for $p_{0}$,

$$
p_{0}(T)=-\frac{3 \Lambda^{2}\left(\kappa K_{2}\right)^{1 / 2}}{2 K_{3}^{2} \nu}+\sqrt{\frac{9 \Lambda^{4} \kappa K_{2}}{4 K_{3}^{4} \nu^{2}}+\frac{\mu_{0}}{\nu}\left(T_{\mathrm{TB}}-T\right)},
$$

the crossover between these two regimes occurs at

$$
\Delta T_{x} \equiv\left(T_{\mathrm{TB}}-T\right)=\frac{9 \Lambda^{4} \kappa K_{2}}{4 K_{3}^{4} \mu_{0} \nu} .
$$

As an aside, this theory can easily be modified to describe a first-order transition between the nematic and $\mathrm{N}_{\mathrm{TB}}$ phases, by changing the fourth-order coefficient $\nu$ to a negative value and adding a sixth-order term to $F_{\text {NTB }}$ in Eq. (1). We have not done so here because the DLS data give no indication of a first-order transition. However, such a modification might be useful for analyzing the nematic$\mathrm{N}_{\mathrm{TB}}$ transition in other systems.

Now that we have determined the ground state, we consider fluctuations about the ground state in the nematic and $\mathrm{N}_{\mathrm{TB}}$ phases.

\section{A. Nematic phase}

In the nematic phase, we must consider fluctuations in the director field about the ground state $\hat{\mathbf{n}}=\hat{\mathbf{z}}$ and fluctuations in the polarization about the ground state $\mathbf{p}=0$. At lowest order, these fluctuations can be described by $\delta \mathbf{n}(\mathbf{r})=\left(n_{x}, n_{y}, 0\right)$ and $\delta \mathbf{p}(\mathbf{r})=\left(p_{x}, p_{y}, p_{z}\right)$. We insert these expressions into the free energy $F_{\mathrm{NTB}}$ [Eq. (1)] and expand to quadratic order in the fluctuating components. We then Fourier transform from position $\mathbf{r}$ to wave vector $\mathbf{q}$, and express the free energy as a quadratic form in $n_{x}(\mathbf{q})$, $n_{y}(\mathbf{q}), p_{x}(\mathbf{q}), p_{y}(\mathbf{q})$, and $p_{z}(\mathbf{q})$,

$$
F=\frac{1}{2} \sum_{\mathbf{q}}\left(\begin{array}{c}
n_{x \mathbf{q}} \\
p_{x \mathbf{q}} \\
n_{y \mathbf{q}} \\
p_{y \mathbf{q}} \\
p_{z \mathbf{q}}
\end{array}\right)^{\dagger}\left(\begin{array}{cc}
K_{1} q_{x}^{2}+K_{2} q_{y}^{2}+K_{3} q_{z}^{2} & -i \Lambda q_{z} \\
i \Lambda q_{z} & \mu+\kappa|\mathbf{q}|^{2} \\
0 & 0 \\
0 & 0 \\
0 & 0
\end{array}\right.
$$

By diagonalizing this quadratic form, we obtain five normal modes:

(1) One hydrodynamic mode is primarily splay-bend director fluctuations, combined with some polarization fluctuations. Its relaxation rate is the ratio of the freeenergy eigenvalue to the relevant viscosity coefficient $\gamma_{n}$, which gives

$$
\Gamma_{1}^{n}=\frac{K_{1} q_{\perp}^{2}+K_{3}^{\mathrm{eff}} q_{z}^{2}}{\gamma_{n}}
$$

in the limit of a long wavelength (small q). Here,

$$
K_{3}^{\mathrm{eff}}=K_{3}-\frac{\Lambda^{2}}{\mu}=K_{3}-\frac{\Lambda^{2}}{\mu_{0}\left(T-T_{0}\right)}
$$

is the renormalized bend elastic constant [11], which shows the effect of coupling the director to the polarization. This effect accounts for the softening of bend fluctuations observed in earlier DLS studies of the director modes when $T \rightarrow T_{\mathrm{TB}}$ from the nematic side [27]. Specifically, Eqs. (7) and (13) imply $K_{3}^{\text {eff }}=0$ at $T=T_{\mathrm{TB}}$.

$$
\left.\begin{array}{ccc}
0 & 0 & 0 \\
0 & 0 & 0 \\
K_{2} q_{x}^{2}+K_{1} q_{y}^{2}+K_{3} q_{z}^{2} & -i \Lambda q_{z} & 0 \\
i \Lambda q_{z} & \mu+\kappa|\mathbf{q}|^{2} & 0 \\
0 & 0 & 2 \eta+\mu+\kappa|\mathbf{q}|^{2}
\end{array}\right)\left(\begin{array}{c}
n_{x \mathbf{q}} \\
p_{x \mathbf{q}} \\
n_{y \mathbf{q}} \\
p_{y \mathbf{q}} \\
p_{z \mathbf{q}}
\end{array}\right) .
$$

(2) Another hydrodynamic mode is primarily twist-bend director fluctuations, combined with some polarization fluctuations. Its relaxation rate is

$$
\Gamma_{2}^{n}=\frac{K_{2} q_{\perp}^{2}+K_{3}^{\mathrm{eff}} q_{z}^{2}}{\gamma_{n}}
$$

again with the renormalized bend elastic constant $K_{3}^{\text {eff }}$.

$(3,4)$ Two nonhydrodynamic modes are mostly polarization fluctuations $p_{x}$ and $p_{y}$, combined with some director fluctuations. In the limit of $\mathbf{q} \rightarrow 0$, these modes have relaxation rate

$$
\Gamma^{p}=\frac{\mu}{\gamma_{p}}=\frac{\mu_{0}\left(T-T_{0}\right)}{\gamma_{p}} .
$$

(5) Another nonhydrodynamic mode is polarization $p_{z}$ by itself. In the limit of $\mathbf{q} \rightarrow 0$, it has relaxation rate

$$
\Gamma^{p^{\prime}}=\frac{2 \eta+\mu}{\gamma_{p^{\prime}}}=\frac{2 \eta+\mu_{0}\left(T-T_{0}\right)}{\gamma_{p^{\prime}}} .
$$

Here, $\gamma_{p}$ and $\gamma_{p^{\prime}}$ are the mode viscosities. 
Overall, we should emphasize the contrast between the nematic phase of the $\mathrm{N}_{\mathrm{TB}}$-forming material studied here and a typical nematic phase. In the $\mathrm{N}_{\mathrm{TB}}$-forming material, we observe a nonhydrodynamic mode with a relaxation rate that decreases with temperature, as the system approaches the transition to the $\mathrm{N}_{\mathrm{TB}}$ phase. The theory attributes this mode to polarization fluctuations, which become less energetically costly as the system develops incipient polar order. By contrast, in a typical nematic phase, no such mode can be observed in DLS experiments; presumably, polarization fluctuations decay too rapidly to be detected.

\section{B. Twist-bend phase}

In the $\mathrm{N}_{\mathrm{TB}}$ phase, the analysis of normal modes is complicated because of the nonuniform, modulated director structure. However, as mentioned in the Introduction, we can simplify this calculation through a coarse-graining approximation, which averages over the director modulation to find the larger-scale properties of the phase. Such coarse-graining has previously been done for the cholesteric phase $[13,14]$, and it shows that the cholesteric has the same macroscopic elastic properties as a smectic phase. In this section, we generalize the coarse-graining procedure to the more complex case of the $\mathrm{N}_{\mathrm{TB}}$ phase. Indeed, it should be an even better approximation for the $\mathrm{N}_{\mathrm{TB}}$ than for the cholesteric phase because the pitch of the $\mathrm{N}_{\mathrm{TB}}$ is so short.

The basic concept of the coarse-graining procedure is illustrated in Fig. 1. We suppose that the director field has a rapid heliconical modulation with respect to a local orthonormal reference frame $\left(\hat{\mathbf{e}}_{1}(\mathbf{r}), \hat{\mathbf{e}}_{2}(\mathbf{r}), \hat{\mathbf{t}}(\mathbf{r})\right)$, and this orthonormal frame varies slowly in space. Furthermore, the heliconical modulation might be displaced upward or downward by a phase $\phi(\mathbf{r})$, which also varies slowly in space. Hence, the director field can be written as

$$
\begin{aligned}
\hat{\mathbf{n}}(\mathbf{r})= & \hat{\mathbf{t}}(\mathbf{r}) \cos \beta+\hat{\mathbf{e}}_{1}(\mathbf{r}) \sin \beta \cos \left(q_{0} z+\phi(\mathbf{r})\right) \\
& +\hat{\mathbf{e}}_{2}(\mathbf{r}) \sin \beta \sin \left(q_{0} z+\phi(\mathbf{r})\right) .
\end{aligned}
$$

In this expression, $\hat{\mathbf{t}}(\mathbf{r})$ is the coarse-grained director, which would be measured in any experiment that averages over the nanoscale heliconical modulation. By analogy with the director field, the polarization field has a rapid helical modulation with respect to the same local orthonormal reference frame, which can be written as

$$
\begin{aligned}
\mathbf{p}(\mathbf{r})= & \hat{\mathbf{e}}_{1}(\mathbf{r}) p_{0} \sin \left(q_{0} z+\phi(\mathbf{r})\right) \\
& -\hat{\mathbf{e}}_{2}(\mathbf{r}) p_{0} \cos \left(q_{0} z+\phi(\mathbf{r})\right)+\delta \mathbf{p}(\mathbf{r}) .
\end{aligned}
$$

Here, $\delta \mathbf{p}(\mathbf{r})=\delta p_{x} \hat{\mathbf{x}}+\delta p_{y} \hat{\mathbf{y}}+\delta p_{z} \hat{\mathbf{z}}$ is a fluctuating additional contribution to the polarization, which varies slowly in space. It is allowed because $\mathbf{p}$ is not restricted to be a unit vector. The contribution $\delta \mathbf{p}(\mathbf{r})$ is the coarse-grained polarization, which would be measured in any experiment that averages over the nanoscale helical modulation.
From Eqs. (17) and (18), we can see that the pseudolayers are surfaces of constant $q_{0} z+\phi(\mathbf{r})=q_{0}(z-u(\mathbf{r}))$, where $u(\mathbf{r})=-\phi(\mathbf{r}) / q_{0}$ is the local pseudolayer displacement. The local helical axis (or pseudolayer normal) is given by the gradient

$$
\hat{\mathbf{N}}(\mathbf{r})=\frac{\boldsymbol{\nabla}\left(q_{0} z+\phi(\mathbf{r})\right)}{\left|\boldsymbol{\nabla}\left(q_{0} z+\phi(\mathbf{r})\right)\right|}=\frac{\hat{\mathbf{z}}-\boldsymbol{\nabla} u}{|\hat{\mathbf{z}}-\boldsymbol{\nabla} u|} .
$$

We now consider the case of a well-aligned sample, as in a light-scattering experiment. In this case, the coarsegrained director $\hat{\mathbf{t}}(\mathbf{r})$ has small fluctuations about $\hat{\mathbf{z}}$, while the phase $\phi(\mathbf{r})$ and coarse-grained polarization $\delta \mathbf{p}(\mathbf{r})$ have small fluctuations around 0 . The full orthonormal reference frame can be written as

$$
\begin{aligned}
\hat{\mathbf{e}}_{1}(\mathbf{r}) & =\left(1-\frac{1}{2} t_{x}^{2}\right) \hat{\mathbf{x}}-\frac{1}{2} t_{x} t_{y} \hat{\mathbf{y}}-t_{x} \hat{\mathbf{z}}, \\
\hat{\mathbf{e}}_{2}(\mathbf{r}) & =-\frac{1}{2} t_{x} t_{y} \hat{\mathbf{x}}+\left(1-\frac{1}{2} t_{y}^{2}\right) \hat{\mathbf{y}}-t_{y} \hat{\mathbf{z}}, \\
\hat{\mathbf{t}}(\mathbf{r}) & =t_{x} \hat{\mathbf{x}}+t_{y} \hat{\mathbf{y}}+\left(1-\frac{1}{2} t_{x}^{2}-\frac{1}{2} t_{y}^{2}\right) \hat{\mathbf{z}},
\end{aligned}
$$

to quadratic order in $t_{x}(\mathbf{r})$ and $t_{y}(\mathbf{r})$. One might think that another variable would be needed to specify the vectors $\hat{\mathbf{e}}_{1}$ and $\hat{\mathbf{e}}_{2}$ in the plane perpendicular to $\hat{\mathbf{t}}$. However, rotations in this plane can be included in the choice of the phase $\phi$. As discussed in Refs. $[13,14]$ for the cholesteric case, such rotations are analogous to gauge transformations. Hence, we make the specific choice of gauge in Eq. (20). With this choice, our orthonormal basis has small fluctuations away from $(\hat{\mathbf{x}}, \hat{\mathbf{y}}, \hat{\mathbf{z}})$.

We insert Eqs. (17) and (18) for the director and polarization fields, together with Eq. (20) for the orthonormal basis, into Eq. (1) for the free energy of the $\mathrm{N}_{\mathrm{TB}}$ phase. We then make the coarse-graining approximation: We integrate over the rapid variations of $\cos q_{0} z$ and $\sin q_{0} z$, assuming that the slowly varying fields are constant over the length scale of the pitch. We thus obtain an effective free energy in terms of the six coarse-grained variables $\phi(\mathbf{r}), t_{x}(\mathbf{r}), t_{y}(\mathbf{r}), \delta p_{x}(\mathbf{r}), \delta p_{y}(\mathbf{r})$, and $\delta p_{z}(\mathbf{r})$. We expand the free energy to quadratic order in these fields and Fourier transform it from position $\mathbf{r}$ to wave vector $\mathbf{q}$ to obtain

$$
F=\frac{1}{2} \sum_{\mathbf{q}}\left(\begin{array}{c}
\phi_{\mathbf{q}} \\
t_{x \mathbf{q}} \\
\delta p_{y \mathbf{q}} \\
t_{y \mathbf{q}} \\
\delta p_{x \mathbf{q}} \\
\delta p_{z \mathbf{q}}
\end{array}\right)^{\dagger} \mathbf{M}(\mathbf{q})\left(\begin{array}{c}
\phi_{\mathbf{q}} \\
t_{x \mathbf{q}} \\
\delta p_{y \mathbf{q}} \\
t_{y \mathbf{q}} \\
\delta p_{x \mathbf{q}} \\
\delta p_{z \mathbf{q}}
\end{array}\right)
$$


Here, $\mathbf{M}(\mathbf{q})$ is a matrix of wave-vector-dependent coefficients, which must be diagonalized to find the normal modes.

It is most convenient to understand the mode structure in the limit of $\mathbf{q} \rightarrow 0$. In this limit, the matrix simplifies to the block-diagonal form

$$
\boldsymbol{M}(0)=\left(\begin{array}{cccccc}
0 & 0 & 0 & 0 & 0 & 0 \\
0 & m_{22} & m_{23} & 0 & 0 & 0 \\
0 & m_{32} & m_{33} & 0 & 0 & 0 \\
0 & 0 & 0 & m_{44} & m_{45} & 0 \\
0 & 0 & 0 & m_{54} & m_{55} & 0 \\
0 & 0 & 0 & 0 & 0 & m_{66}
\end{array}\right),
$$

where

$$
\begin{aligned}
& m_{22}=m_{44}=p_{0} q_{0} \Lambda \sin \beta+\frac{1}{2}\left(K_{1}+K_{2}-2 K_{3}\right) q_{0}^{2} \sin ^{2} \beta, \\
& m_{33}=m_{55}=\mu+2 \nu p_{0}^{2}+\eta \sin ^{2} \beta, \\
& m_{23}=m_{32}=-m_{45}=-m_{54}=-\frac{1}{2} q_{0} \Lambda \sin ^{2} \beta \\
& m_{66}=(2 \eta+\mu)+\nu p_{0}^{2}-2 \eta \sin ^{2} \beta .
\end{aligned}
$$

From this block-diagonal form, we can extract the following six normal modes:

(1) The phase $\phi=-u / q_{0}$ is itself a normal mode. This mode is hydrodynamic, with zero energy (and zero relaxation rate) in the limit of $\mathbf{q} \rightarrow 0$. It is analogous to the layer displacement of a smectic-A phase, which costs zero energy for uniform displacement. It is also analogous to the hydrodynamic director mode in a cholesteric phase (which is called the pure twist mode in the theory of cholesteric light scattering [28]). It is visualized in terms of pseudolayers in Figs. 8(a) and 8(b).

$(2,3)$ The coarse-grained director tilt $t_{x}$ and polarization $\delta p_{y}$ are coupled by the helicity of the $\mathrm{N}_{\mathrm{TB}}$ phase. Together, they form a pair of normal modes, both of which are nonhydrodynamic, with nonzero energy (and nonzero relaxation rate) in the limit of $\mathbf{q} \rightarrow 0$. In the limit of weak coupling, which is given by the criterion $m_{22} m_{33} \gg m_{23}^{2}$,

$$
\begin{gathered}
\Gamma^{t}=m_{22} / \gamma_{t}, \\
\Gamma^{p}=m_{33} / \gamma_{p} .
\end{gathered}
$$

Here, $\gamma_{t}$ and $\gamma_{p}$ are phenomenological viscosities associated with the normal modes. The two modes are analogous to tilt and polarization fluctuations in a chiral smectic-A phase. The tilt mode is also analogous to the nonhydrodynamic director mode in a cholesteric phase (which is called the umbrella mode in the theory of cholesteric light scattering [28]). The tilt mode is visualized in Fig. 8(c); the polarization mode is not visualized.
The coupling between tilt and polarization in these modes is the electroclinic effect, which was previously predicted and observed in Ref. [10]. In this effect, a polarization $\delta p_{y}$ induces a tilt $t_{x}$, and vice versa, as in a chiral smectic-A phase. The sign of the electroclinic effect depends on the sign of the coupling $m_{23}$, which is controlled by the sign of the helicity $q_{0}$. For that reason, domains of right- and left-handed helicity must have opposite electroclinic effects. The previous work of Ref. [10] presented one derivation of this effect; here, we see that our theoretical formalism provides an alternative derivation of the same effect.

$(4,5)$ The coarse-grained director tilt $t_{y}$ and polarization $\delta p_{x}$ form another pair of nonhydrodynamic normal modes, which is degenerate with the previous pair.

(6) The polarization component $\delta p_{z}$ is itself a nonhydrodynamic normal mode. Its relaxation rate is

$$
\Gamma^{p^{\prime}}=m_{66} / \gamma_{p^{\prime}},
$$

where $\gamma_{p^{\prime}}$ is the viscosity of this mode.

If the wave vector $\mathbf{q}$ is small but nonzero, the five nonhydrodynamic modes are only slightly changed. To model their relaxation rates, we can still use Eqs. (24) and (25) derived above. However, the hydrodynamic mode is more significantly changed. We can consider the cases of q parallel and perpendicular to the $z$ direction separately.

For $\mathbf{q}$ in the $z$ direction, the hydrodynamic mode still involves the phase $\phi$ by itself, not coupled with any other coarse-grained degrees of freedom. This mode is visualized in Fig. 8(d). It is a $z$-dependent rotation of the heliconical director field $\hat{\mathbf{n}}(\mathbf{r})$, which does not change the coarsegrained director $\hat{\mathbf{t}}$. Equivalently, this mode can be regarded as a $z$-dependent displacement $u=-\phi / q_{0}$ of the pseudolayers, leading to alternating compression and dilation of the pseudolayer structure. In the limit of long wavelength (small q), the free-energy cost of this fluctuation is $\frac{1}{2} B_{\text {eff }} q_{z}^{2}\left|u_{\mathbf{q}}\right|^{2}$, where

$$
B_{\text {eff }}=\left(K_{2} \sin ^{4} \beta+K_{3} \sin ^{2} \beta \cos ^{2} \beta+\kappa p_{0}^{2}\right) q_{0}^{2} .
$$

Hence, the relaxation rate is $\Gamma^{u}\left(q_{z}\right)=\frac{1}{2} \gamma_{u}^{-1} B_{\text {eff }} q_{z}^{2}$, where $\gamma_{u}$ is the relevant viscosity.

For $\mathbf{q}$ in the $x$ direction, the hydrodynamic normal mode is a linear combination of $\phi, t_{x}$, and $\delta p_{y}$, as visualized in Fig. 8(e). This mode is an $x$-dependent rotation of the $\hat{\mathbf{n}}(\mathbf{r})$ or, equivalently, an $x$-dependent displacement of the pseudolayers, leading to curvature of the pseudolayer structure. This displacement is accompanied by a tilt of the coarse-grained director in the $x$ direction, so the local $\hat{\mathbf{t}}$ remains normal to the local pseudolayers. If $\mathbf{q}$ is in any other direction in the $(x, y)$ plane, the same description applies with the corresponding rotation. The free-energy cost of this fluctuation is $\frac{1}{2} K_{\text {eff }} q_{\perp}^{4}\left|u_{\mathbf{q}}\right|^{2}$, where 
(a) Ground state

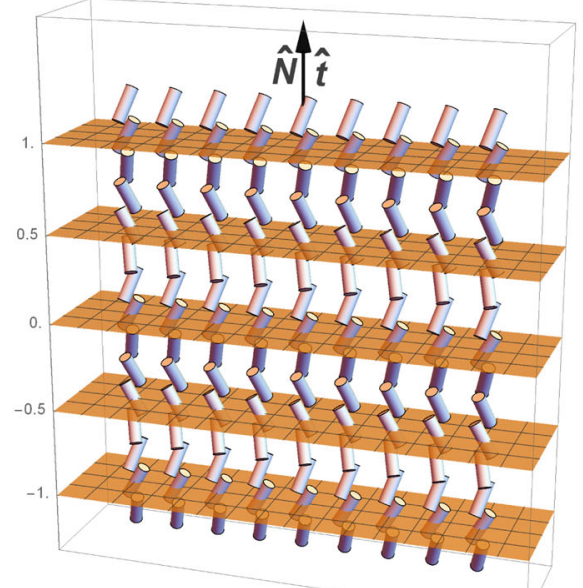

(b) Hydrodynamic mode $q=0$

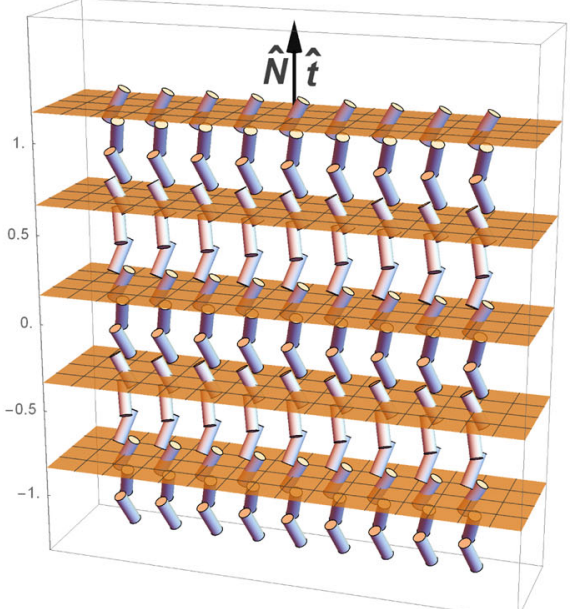

(c) Tilt mode $q=0$

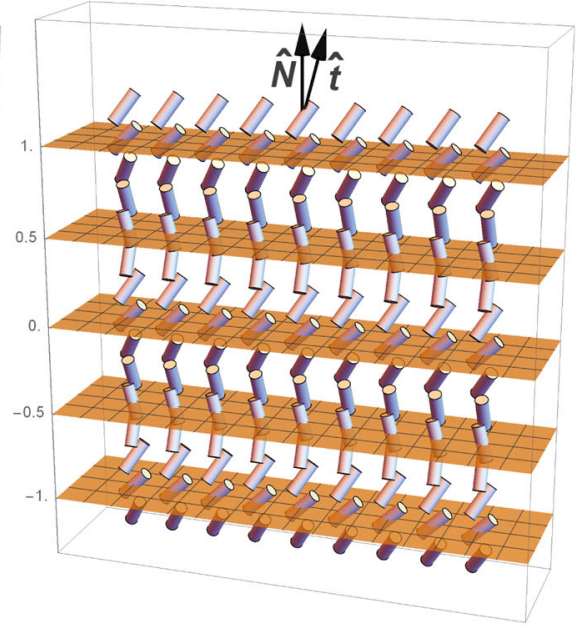

$\begin{array}{lll}\text { (d) Hydrodynamic mode } q=q_{z} \hat{z} & \text { (e) Hydrodynamic mode } q=q_{\perp} \hat{x}\end{array}$
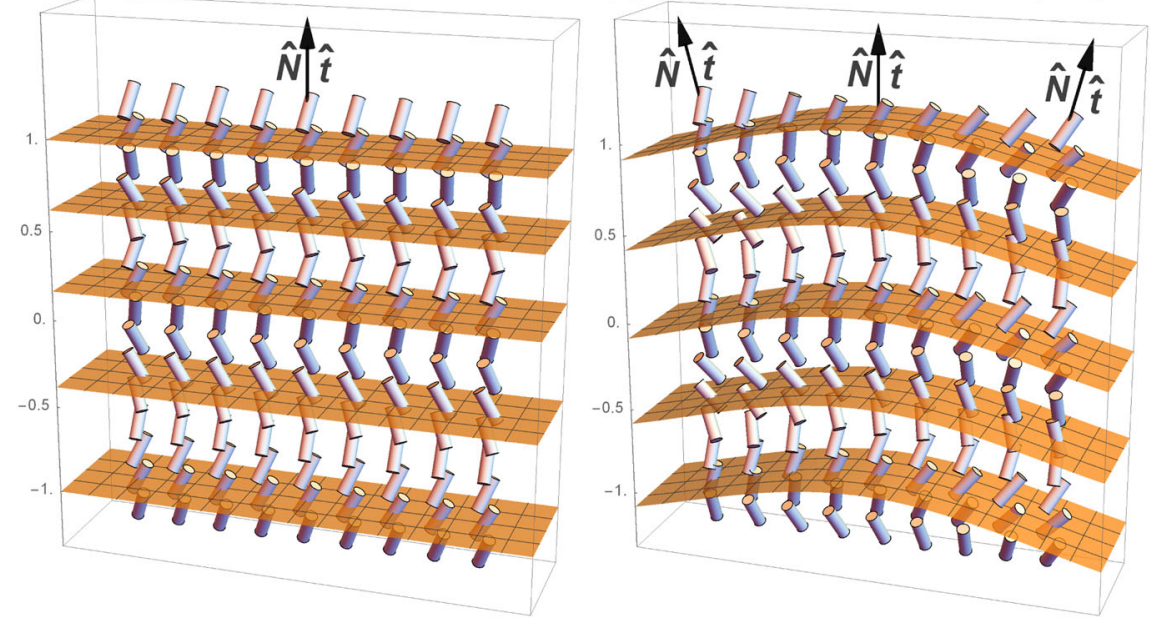

FIG. 8. Visualization of certain fluctuation modes in the $\mathrm{N}_{\text {TB }}$ phase [29]. Small cylinders represent the heliconical director field $\hat{\mathbf{n}}(\mathbf{r})$, and surfaces represent the pseudolayers. (a) Ground state. (b) Hydrodynamic mode with wave vector $\mathbf{q}=0$, with uniform rotation of $\hat{\mathbf{n}}(\mathbf{r})$ and hence uniform displacement of pseudolayers; this mode has no energy cost with respect to the ground state. (c) Nonhydrodynamic tilt mode, with the coarse-grained director $\hat{\mathbf{t}}$ [average of $\hat{\mathbf{n}}(\mathbf{r})$ ] tilted with respect to pseudolayer normal. (d) Hydrodynamic mode with $\mathbf{q}=q \hat{\mathbf{z}}$, with $z$-dependent rotation of $\hat{\mathbf{n}}(\mathbf{r})$ and $z$-dependent displacement of pseudolayers (leading to compression and dilation). (e) Hydrodynamic mode with $\mathbf{q}=q \hat{\mathbf{x}}$, with $x$-dependent rotation of $\hat{\mathbf{n}}(\mathbf{r})$ and $x$-dependent displacement of pseudo-layers (leading to curvature), accompanied by tilt so that $\hat{\mathbf{t}}$ remains normal to pseudolayers.

$$
K_{\text {eff }}=K_{1} \cos ^{2} \beta+\frac{1}{2} K_{3} \sin ^{2} \beta+\frac{1}{2} \kappa p_{0}^{2},
$$

to lowest order in small $\beta$. Hence, the relaxation rate is $\Gamma^{u}\left(q_{\perp}\right)=\frac{1}{2} \gamma_{u}^{-1} K_{\text {eff }} q_{\perp}^{4}$.

The effective elastic constant $D_{\text {eff }}$ for tilt of the coarsegrained director $\hat{\mathbf{t}}$ away from the pseudolayer normal $\hat{\mathbf{N}}$ [Eq. (14)] follows from the earlier discussion of the nonhydrodynamic modes in the $\mathbf{q}=0$ limit: $D_{\text {eff }}=$ $m_{22}=m_{44}$. For small $p_{0}$ and $\beta, \Lambda p_{0} / K_{3} \approx q_{0} \sin \beta$ from Eq. (4), and therefore

$$
D_{\text {eff }} \approx \frac{1}{2}\left(K_{1}+K_{2}\right) \sin ^{2} \beta q_{0}^{2} \approx \frac{\left(K_{1}+K_{2}\right)}{2 K_{3}^{2}} \Lambda^{2} p_{0}^{2} .
$$

The first expression for $D_{\text {eff }}$ above matches that calculated from the coarse-graining approach of Ref. [17], and, in addition, for small $\beta$ and polarization elastic constant $\kappa$, the expressions for $B_{\text {eff }}$ and $K_{\text {eff }}$ [Eqs. (26) and (27)] are also consistent with Ref. [17].

Thus, the effective elasticity associated with the pseudolayered $\mathrm{N}_{\mathrm{TB}}$ phase is equivalent to a smectic-A phase, with $B_{\text {eff }}, K_{\text {eff }}$, and $D_{\text {eff }}$ playing the roles of the elastic moduli for compression and bending of the smectic layers, and for tilt of the director away from the layer normal, respectively. In that way, coarse-graining of the $\mathrm{N}_{\mathrm{TB}}$ phase is analogous to earlier work on coarse-graining of the cholesteric phase, which also has effective smectic elasticity [13,14]. 
On the other hand, it is important not to overplay the analogy, especially as far as details of the pretransitional temperature dependence of elastic constants are concerned. We will return to this point at the end of the next section.

\section{DISCUSSION}

We can now compare the calculated normal modes with the light-scattering experiment.

\section{A. Nematic phase}

The fluctuating part of the dielectric tensor can be expressed in terms of the normal modes using the relation

$$
\epsilon_{i j}(\mathbf{r})=\Delta \epsilon^{n} n_{i} n_{j}+\Delta \epsilon_{\mathrm{sat}}^{p} p_{i} p_{j}
$$

where $(i, j)=(x, y, z), \Delta \epsilon^{n}$ is the dielectric anisotropy associated with the orientational ordering of $\hat{\mathbf{n}}$, and $\Delta \epsilon_{\text {sat }}^{p}$ is the saturated value of the dielectric anisotropy associated with the $\mathbf{p}$ ordering.

In geometry G1, with $q_{z}=0$, the fluctuations in $\hat{\mathbf{n}}$ and $\mathbf{p}$ decouple. The former yield the usual pair of hydrodynamic director modes $\left(n_{1}, n_{2}\right)$, while the latter produce a doubly degenerate nonhydrodynamic mode associated with $p_{x}, p_{y}$, plus an independent nonhydrodynamic mode associated with $p_{z}$. Assuming large coefficient $\eta$ in Eq. (1), we can neglect $p_{z}$. Since the incident polarization in geometry G1 is along $\hat{\mathbf{z}}$, the relevant elements of $\epsilon_{i j}$ for depolarized scattering are $\epsilon_{x z}$ and $\epsilon_{y z}$. Assuming negligible $p_{z}$, these elements are dominated by the director modes, and specifically in our experiment for large $\theta_{s}$, by the splay fluctuations in the normal mode $n_{1}$. Therefore, in agreement with our experimental results for geometry G1, the model with large $\eta$ predicts that the DLS correlation function is described by a single exponential decay (with relaxation rate $\Gamma_{1}^{n}$ ) and that the contribution from nonhydrodynamic polarization fluctuations is not observable.

The situation is different in geometry G2, where $\mathbf{q}=$ $q_{x} \hat{\mathbf{x}}+q_{z} \hat{\mathbf{z}}$ for scattering in the $x-z$ plane. (The choice of $x-z$ or $y-z$ is arbitrary.) In depolarized DLS, with the incident light polarized along $\hat{\mathbf{y}}$, we probe fluctuations $\epsilon_{z y}$ and $\epsilon_{x y}$. From the former, we expect and observe the $n_{2}$ (twist-bend) hydrodynamic mode. The latter $\left(\epsilon_{x y}\right)$ couples to nonhydrodynamic polarization fluctuations transverse to the nematic ordering axis, which contribute maximally to the DLS signal in the "dark director" limit of G2, where $\epsilon_{z y} \rightarrow 0$ and $\epsilon_{x y}$ dominates.

Since $\epsilon_{x y}$ is quadratic in $\mathbf{p}$ [Eq. (29)], the time correlation function $\left\langle\epsilon_{x y}^{*}(0) \epsilon_{x y}(\tau)\right\rangle$, relevant for DLS, contains the higher-order correlation function $C\left(p_{x}, p_{y}\right)=\left\langle p_{x}^{*}(0) p_{y}^{*}(0)\right.$ $\left.p_{x}(\tau) p_{y}(\tau)\right\rangle$ of the polarization fluctuations. (Here, $\tau=$ delay time). Based on the structure of the free energy written in terms of $n_{1}, n_{2}, p_{x}$, and $p_{y}$, and assuming the fluctuations are Gaussian random variables with zero mean, $C\left(p_{x}, p_{y}\right)$ can be reduced to $\left\langle p_{x}^{*}(0) p_{x}(\tau)\right\rangle\left\langle p_{y}^{*}(0) p_{y}(\tau)\right\rangle$.

In the limit that the energy associated with $\mathbf{p}$ fluctuations is much greater than that of the $\mathbf{p}-\hat{\mathbf{n}}$ coupling, and that the latter is much greater than the elastic energy of $\hat{\mathbf{n}}$ fluctuations, the correlation function is a well-separated double exponential decay, as observed in our experiment [30], with the faster decay characterized by relaxation rate $\Gamma_{2}^{p} \sim$ (constant in $q$ ) for the $\mathbf{p}$ fluctuations, and the slower decay characterized by a rate $\Gamma_{2}^{n} \sim q^{2}$ representing a mixture of director modes. This mixture could explain the broadening of the slower decay indicated by our data analysis.

Outside of the dark director geometry, the twist-bend director scattering from $\epsilon_{z y}$, which is linear in $n_{2}$, prevails, and the fast decay makes only a weak contribution to the DLS correlation function-again in agreement with the experiment. The relaxation rate of the director mode (Fig. 6) decreases as $T \rightarrow T_{\mathrm{TB}}$ from above, but only by a factor of about 1.6. This modest decrease remains consistent with the expected softening of bend fluctuations, $K_{3}^{\text {eff }} \rightarrow 0$ as $T \rightarrow T_{\mathrm{TB}}$ [see Eqs. (7) and (11)] since Eq. (12) indicates that the relaxation rate $\Gamma_{2}^{n} \simeq K_{2} q_{\perp}^{2} / \gamma_{n}$ for the condition $q_{\perp}^{2} \gg q_{z}^{2}$ in geometry G2. Thus, in the scattering geometry used, $\Gamma_{2}^{n}$ is not very sensitive to the temperature dependence of $K_{3}^{\text {eff }}$.

\section{B. Twist-bend phase}

The spectrum of modes is related to fluctuations of the dielectric tensor through a modified version of Eq. (29), where $\Delta \epsilon^{n} n_{i} n_{j}$ is replaced by $\Delta \epsilon^{t} t_{i} t_{j}$. The hydrodynamic mode is the extension into the $\mathrm{N}_{\text {Tв }}$ phase of the splay-bend director mode $n_{1}$, which is observed in the nematic phase in geometry G1. The nonhydrodynamic tilt mode is the extension of the hydrodynamic twist-bend director mode $n_{2}$, which is observed in the nematic phase in geometry G2. It acquires a large energy gap when the heliconical structure forms, analogous to the gap in $n_{2}$ that develops in a smectic-A phase due to the large energy cost of tilting the director away from the layer normal. The coarse-grained model thus accounts for both the slow hydrodynamic mode [data labeled (b) in the bottom panel of Fig. 4] and the slower of the pair of nonhydrodynamic modes [data labeled (a) in the bottom panel of Fig. 4], which are observed in experimental geometries G1 and G2, respectively.

The faster nonhydrodynamic mode in the $\mathrm{N}_{\mathrm{TB}}$ phase is detected in the dark director limit of geometry G2 [see correlation data labeled (c) in the bottom panel of Fig. 4]. As in the nematic case, it can be associated with fluctuations of the polarization $\left(\delta p_{x}, \delta p_{y}\right)$. Because the polarization fluctuations are only observed for a scattering geometry where the $\hat{\mathbf{t}}$ fluctuations are dark, the coupling between tilt and polarization fluctuations must be weak.

The coarse-grained theory predicts additional terms in the expression for the energy gap of these fluctuations in 
the $\mathrm{N}_{\mathrm{TB}}$ phase compared with the nematic phase [see Eq. (24b) compared with Eq. (15)]. These terms imply an increase in the relaxation rate $\Gamma_{p}$ of the polarization mode at $T_{\mathrm{TB}}$, which is consistent with the experimentally observed behavior (Fig. 6). According to the model, this increase in $\Gamma_{p}$ signals a transition to a heliconical structure with $\beta \neq 0$ and $p_{0} \neq 0$.

The slow relaxation process that mixes with the fast polarization fluctuations in the correlation function is also explained by the theory: When $q_{z}$ and $q_{\perp}$ are nonzero, as is generally the case in the G2 geometry, $\delta p_{x}$ and $\delta p_{y}$ mix with the slow hydrodynamic variable $\phi$ and with $\hat{\mathbf{t}}$, and thus the correlation function contains a slow component corresponding to undulation of the pseudolayers and splay of $\hat{\mathbf{t}}$.

The final nonhydrodynamic mode predicted by the theory, related to $\delta p_{z}$, has an even higher relaxation rate, which is not detected in our experiment. This high relaxation rate implies a relatively large value for the coefficient $\eta$ in the free energy of Eq. (1).

To fit the experimental data for relaxation rates as functions of temperature, we combine Eq. (15) in the nematic phase and Eqs. (23) and (24) in the $\mathrm{N}_{\mathrm{TB}}$ phase. For the equilibrium cone angle $\beta$ and pitch wave number $q_{0}$, we use the leading terms in Eqs. (4) and (5) near the second-order transition, which give $\sin ^{2} \beta \approx p_{0}\left(\kappa / K_{2}\right)^{1 / 2}$ and $q_{0} \approx\left(\Lambda / K_{3}\right) p_{0}^{1 / 2}\left(K_{2} / \kappa\right)^{1 / 4}$. For the equilibrium polarization $p_{0}$, we use Eq. (9), which applies when $T<T_{\mathrm{TB}}-\Delta T_{x}$ [see Eq. (10)]; the validity of this condition is confirmed below. The predicted relaxation rates then become

$$
\begin{aligned}
& \Gamma^{p}\left(T>T_{\mathrm{TB}}\right)=\frac{\Lambda^{2}}{K_{3} \gamma_{p}}+\frac{\mu_{0}}{\gamma_{p}}\left(T-T_{\mathrm{TB}}\right), \\
& \Gamma^{t}\left(T<T_{\mathrm{TB}}\right)= \frac{\Lambda^{2}\left(K_{1}+K_{2}\right) \mu_{0}\left(T_{\mathrm{TB}}-T\right)}{2 \gamma_{t} K_{3}^{2} \nu}, \\
& \Gamma^{p}\left(T<T_{\mathrm{TB}}\right)= \frac{\Lambda^{2}}{K_{3} \gamma_{p}}+\frac{\eta}{\gamma_{p}} \sqrt{\frac{\kappa \mu_{0}\left(T_{\mathrm{TB}}-T\right)}{K_{2} \nu}} \\
&+\frac{\mu_{0}}{\gamma_{p}}\left(T_{\mathrm{TB}}-T\right) .
\end{aligned}
$$

Generally, the orientational viscosities $\gamma_{t}$ and $\gamma_{p}$ each have an activated (Arrhenius) temperature dependence of the form $\gamma=\gamma_{0} \exp \left(T_{A} / T\right)$, where $T_{A}$ is an activation temperature, and $T, T_{A}$ are measured in Kelvin. Since the experimental data for $\Gamma^{p}\left(T>T_{\mathrm{TB}}\right)$ and $\Gamma^{t}\left(T<T_{\mathrm{TB}}\right)$ show a linear temperature dependence, it is reasonable to expand $\gamma$ around $T_{\mathrm{TB}}$ to linear order in $T-T_{\mathrm{TB}}$. This results in the following expression for $\Gamma^{p}$ to lowest order in $\left|T-T_{\mathrm{TB}}\right|$ and for large $\eta$,

$$
\begin{aligned}
& \Gamma^{p}\left(T>T_{\mathrm{TB}}\right) \approx \frac{\Lambda^{2}}{K_{3} \gamma_{p}\left(T_{\mathrm{TB}}\right)}+\frac{\tilde{\mu}_{0}}{\gamma_{p}\left(T_{\mathrm{TB}}\right)}\left(T-T_{\mathrm{TB}}\right), \\
& \Gamma^{p}\left(T<T_{\mathrm{TB}}\right) \approx \frac{\Lambda^{2}}{K_{3} \gamma_{p}\left(T_{\mathrm{TB}}\right)}+\frac{\eta}{\gamma_{p}\left(T_{\mathrm{TB}}\right)} \sqrt{\frac{\kappa \mu_{0}}{K_{2} \nu}} \sqrt{T-T_{\mathrm{TB}}},
\end{aligned}
$$

where $\tilde{\mu}_{0}=\mu_{0}\left(1+\Lambda^{2} T_{A} / K_{3} \mu_{0} T_{\mathrm{TB}}^{2}\right)$ and $\gamma_{p}\left(T_{\mathrm{TB}}\right)$ is the value of $\gamma_{p}$ at the transition. Because the approximately $10-\mathrm{K}$ range over which we measured $\Gamma^{t}$ is small compared to $T_{\mathrm{TB}}=367.4 \mathrm{~K}$, we may reasonably approximate $\gamma_{t}(T)$ in Eq. (30b) by $\gamma_{t}\left(T_{\mathrm{TB}}\right)$.

We compare Eqs. (30b) and Eq. (31) with the data in Fig. 6 as follows: First, fitting Eq. (31a) to the data for $\Gamma^{p}$ in the nematic phase, we find $\tilde{\mu}_{0} / \gamma_{p}\left(T_{\mathrm{TB}}\right)=3600 \mathrm{~s}^{-1} \mathrm{~K}^{-1}$ and $\Lambda^{2} / K_{3} \gamma_{p}\left(T_{\mathrm{TB}}\right)=1.1 \times 10^{5} \mathrm{~s}^{-1}$, so $\Lambda^{2} / K_{3} \tilde{\mu}_{0}=30 \mathrm{~K}$. The fit is shown as a solid line in Fig. 6 (bottom panel, $T>T_{\mathrm{TB}}$ ).

Second, the data for $\Gamma^{t}$ in the $\mathrm{N}_{\text {Тв }}$ phase are consistent with the linear dependence in Eq. (30b). This consistency confirms that the experiment is in the regime where $p_{0}$ follows the approximation of Eq. (9) rather than Eq. (8). The experimental slope corresponds to the combination of parameters $\Lambda^{2}\left(K_{1}+K_{2}\right) \mu_{0} / 2 \gamma_{t}\left(T_{\mathrm{TB}}\right) K_{3}^{2} \nu=84000 \mathrm{~s}^{-1} \mathrm{~K}^{-1}$. This fit is shown as a solid line in Fig. 6 (top panel, $T<T_{\mathrm{TB}}$ ).

Third, the data for $\Gamma^{p}$ in the $\mathrm{N}_{\mathrm{TB}}$ phase can be fit to the expression in Eq. (31b), as shown by the solid line in Fig. 6 (bottom panel, $T<T_{\mathrm{TB}}$ ). In this fit, we fix the parameter $\Lambda^{2} / K_{3} \gamma_{p}\left(T_{\mathrm{TB}}\right)$ to the value obtained from the analysis of $\Gamma^{p}$ in the nematic phase. The resulting one-parameter fit yields $\eta\left(\kappa \mu_{0}\right)^{1 / 2}\left(K_{2} \nu\right)^{-1 / 2} / \gamma_{p}\left(T_{\mathrm{TB}}\right)=4.3 \times 10^{6} \mathrm{~K}^{-1 / 2} \mathrm{~s}^{-1}$.

We now combine the last fit result with two estimates. First, estimating $\sin ^{2} \beta \simeq 0.015$ from the optical birefringence data in Ref. [5] at $T_{\mathrm{TB}}-T \simeq 1 \mathrm{~K}$ and also taking $p_{0} \simeq 0.1$ at this temperature, we get from Eq. (5) (for small в) $\sqrt{\kappa / K_{2}} \approx \sin ^{2} \beta / p_{0} \simeq 0.15$, and then from Eq. (9), $\left(\mu_{0} / \nu\right)^{1 / 2} \simeq 0.1 \mathrm{~K}^{-1 / 2}$. Together with the fit results, these estimates give $\eta / \tilde{\mu}_{0} \simeq 10^{5} \mathrm{~K}$. This large value indicates that the relaxation rate $\Gamma^{p^{\prime}}$ of longitudinal polarization fluctuations in Eqs. (16) and (25) is much larger than $\Gamma^{p}$, and hence explains why those fluctuations are not observed in our experiment.

We may also verify two conditions on which our analysis is predicated: (1) that $T_{\mathrm{TB}}-T>\Delta T_{x}=9 \Lambda^{4} \kappa K_{2} /$ $\left(4 K_{3}^{4} \mu_{0} \nu\right)$ [see Eq. (10) and accompanying discussion above] over the temperature range of our data in the $\mathrm{N}_{\mathrm{TB}}$ phase, meaning Eq. (9) applies, and therefore Eq. (30b) is valid; and (2) that $m_{22} m_{33} \gg m_{23}^{2}$, which validates the decoupling approximation for the polarization and tilt modes, and hence the use of Eqs. (30b) and (30c) for their relaxation rates. 
First, using the numerical results above and assuming $\mu_{0} \simeq \tilde{\mu}_{0}$, we estimate $\Delta T_{x} \simeq 0.3 \mathrm{~K}$, and thus confirm that our $\mathrm{N}_{\mathrm{TB}}$ data are strictly in the regime $T_{\mathrm{TB}}-T \gg \Delta T_{x}$ where Eq. (9) applies. Next, from Eqs. (4) and (5) for small $\sin ^{2} \beta$, we have $\sin ^{2} \beta \approx p_{0} \sqrt{\kappa / K_{2}}$ and $q_{0} \approx \sqrt{p_{0}}\left(\Lambda / K_{3}\right)$ $\left(K_{2} / \kappa\right)^{1 / 4}$. Equations $(23)$ then imply $m_{22} \approx\left(\Lambda p_{0} / K_{3}\right)^{2}$ $\left(K_{1}+K_{2}\right) / 2, m_{33}>\eta p_{0} \sqrt{\kappa / K_{2}}$, and $m_{23}=-\left(\Lambda^{2} / 2 K_{3}\right)$ $\left(\kappa / K_{2}\right)^{1 / 4} p_{0}^{3 / 2}$. Thus, the condition $m_{22} m_{33} \gg m_{23}^{2}$ may be written as $\eta / \mu_{0} \gg\left(\Lambda^{2} /\left(2 K_{3} \mu_{0}\right)\right)\left(K_{3} /\left(K_{1}+K_{2}\right)\right)$. Again assuming $\mu_{0} \simeq \tilde{\mu}_{0}$ and using the parameters from the fits given above, this reduces to $10^{5} \mathrm{~K} \gg 15 \mathrm{~K}\left(K_{3} /\left(K_{1}+K_{2}\right)\right)$, which is clearly valid.

Now let us turn to the data for the inverse scattering intensity $I_{2}^{-1}$ in Fig. 7. Recall that these data were recorded in geometry G2 for $\theta_{i}=15^{\circ}, \theta_{s}=40^{\circ}$, where the scattering is dominated by optic axis fluctuations (i.e., $\hat{\mathbf{n}}$ or $\hat{\mathbf{t}}$ ). In each phase, $I_{2}^{-1}$ is proportional to the free-energy density of these fluctuations. On this basis, we can make two useful comparisons between experiment and theory:

(1) Since $I_{2}^{-1} \propto \gamma_{t} \Gamma^{t}$ in the $\mathrm{N}_{\mathrm{TB}}$ phase, and since $\Gamma^{t}$ is essentially linear in $T_{\mathrm{TB}}-T$ (Fig. 6), we expect and observe the same for $I_{2}^{-1}$ (Fig. 7).

(2) In the nematic phase, the free-energy density of director fluctuations is given by $\frac{1}{2} K_{2} q_{\perp}^{2}$ (from the Frank free energy with the experimental condition $K_{2} q_{\perp}^{2} \gg K_{3} q_{z}^{2}$ appropriate for geometry $\mathrm{G} 2$ ). In the $\mathrm{N}_{\mathrm{TB}}$ phase, the freeenergy density of coarse-grained director fluctuations is given by $\frac{1}{2}\left(K_{1}+K_{2}\right) q_{0}^{2} \sin ^{2} \beta$ [from Eq. (24a) for $\gamma_{t} \Gamma^{t}$ combined with the result $p_{0} \approx\left(K_{3} / \Lambda\right) q_{0} \sin \beta$ near the transition]. Hence, the ratio of scattering intensities in the two phases should be

$$
\frac{I_{2}\left(T>T_{\mathrm{TB}}\right)}{I_{2}\left(T<T_{\mathrm{TB}}\right)} \approx \frac{\left(K_{1}+K_{2}\right) q_{0}^{2} \sin ^{2} \beta}{2 K_{2} q_{\perp}^{2}} \approx \frac{q_{0}^{2} \sin ^{2} \beta}{q_{\perp}^{2}} .
$$

From Ref. [5], using relative values of the optical birefringence at $T=T_{\mathrm{TB}}$ and $T-T_{\mathrm{TB}}=-5^{\circ} \mathrm{C}$, we estimate $\beta=7.5^{\circ}$. From the same reference, FFTEM textures show that the pitch is $2 \pi / q_{0}=9.3 \mathrm{~nm}$. In our experimental geometry, $q_{\perp}=2 \pi\left(\sin \theta_{i}+\sin \theta_{s}\right) / \lambda=0.011 \mathrm{~nm}^{-1}$. Combining these numbers gives

$$
\frac{I_{2}\left(T>T_{\mathrm{TB}}\right)}{I_{2}\left(T<T_{\mathrm{TB}}\right)} \approx 70 .
$$

By comparison, the experimental intensity ratio in Fig. 7 (between the nematic phase just above the transition and the $\mathrm{N}_{\mathrm{TB}}$ in the middle of its range, $5^{\circ} \mathrm{C}$ below the transition) is approximately 60 . This quantitative similarity gives additional support to the theory.

According to Eqs. (28), the effective elastic constant $D_{\text {eff }}$ (which penalizes fluctuations of the coarse-grained director against the pseudolayer normal in the $\mathrm{N}_{\mathrm{TB}}$ phase) is proportional to $p_{0}^{2}$ and thus, by Eq. (9), to $T_{\mathrm{TB}}-T$. Since $I_{2}^{-1} \propto D_{\text {eff }}$, the linear temperature dependence of the data in Fig. 7 confirms that the pretransitional behavior of $D_{\text {eff }}$ is described by mean-field theory. However, this is not the case in the analogous smectic-A system near the transition to the nematic phase (temperature $T_{N A}$ ). Here, experiment indicates $D \propto\left(T_{N A}-T\right)^{x}$ with $x \approx 0.5$ [31-33], whereas mean-field theory predicts $x=1$, and a more sophisticated, anisotropic scaling analysis gives $x \approx 0.67$ [28].

Finally, we note that numerous works [31-36] over the years have reported on the temperature dependence of the smectic-layer compression constant $B$ as $T \rightarrow T_{N A}$ (from below). However, to our knowledge, no data are yet available for the $T$ dependence of the analogous quantity, $B_{\text {eff }}$, as $T \rightarrow T_{\mathrm{TB}}$ [37] in the $N_{\mathrm{TB}}$ phase.

\section{CONCLUSION}

Our DLS study of a twist-bend nematic liquid crystal demonstrates the presence of a pair of temperaturedependent, nonhydrodynamic fluctuation modes connected to the $\mathrm{N}_{\mathrm{TB}}$ structure. One of these modes is associated with twist-bend director fluctuations in the presence of a shortpitch heliconical modulation of $\hat{\mathbf{n}}$, while the other is accounted for by fluctuations in a vector order parameter that corresponds to a helical polarization field coupled to the director modulation. The behavior of both modes, as well as the presence of a single hydrodynamic mode in the $\mathrm{N}_{\mathrm{TB}}$ phase (associated with splay fluctuations of the helical pitch axis), is quantitatively explained by a theoretical model based on two components: (1) a Landau-de Gennes freeenergy density, which is expanded in the director and polarization fields, and (2) a coarse-graining of this free energy that analogizes the heliconical structure to a smecticA-like system characterized by a pseudolayer displacement field and an effective director normal to the layers. The model specifically describes how the distortions of the pseudolayers couple to the polarization field-a result useful not only to interpret our DLS measurements but also, as reported very recently [38], to account for second harmonic generation from defects in the $\mathrm{N}_{\mathrm{TB}}$ pseudolayer structure.

For the future, it would be interesting to determine the effective elastic constant $\left(B_{\text {eff }}\right)$ for pseudolayer compression as a function of temperature. The ratio $B_{\text {eff }} / K_{\text {eff }} \approx B_{\text {eff }} / K_{1}$ can, for instance, be measured by DLS. It could also be illuminating to probe the response of polarization fluctuations to an applied electric field. Finally, extending the Landau-de Gennes theory to include a first-order N- $\mathrm{N}_{\mathrm{TB}}$ transition may prove useful for understanding experimental results on a wider range of dimers or monomer-dimer mixtures.

\section{ACKNOWLEDGMENTS}

We are grateful to S. Pardaev for assistance in collecting the light-scattering data. We thank the following agencies 
for support: the NSF under Grants No. DMR-1307674 (Z.P., J. G., A. J., S.S.), No. DMR-1409658 (S. M. S., J. V.S.), and No. DMR-1410378 (V. B., O. D. L.); the EPSRC under Grant No. EP/J004480/1 (G. M. and C. W.); and the EU under Project No. 216025 (M. G. T.).

[1] D. Chen, J. H. Porada, J. B. Hooper, A. Klittnick, Y. Shen, M. R. Tuchband, E. Korblova, D. Bedrov, D. M. Walba, M. A. Glaser, J.E. Maclennan, and N. A. Clark, Chiral Heliconical Ground State of Nanoscale Pitch in a Nematic Liquid Crystal of Achiral Molecular Dimers, Proc. Natl. Acad. Sci. U.S.A. 110, 15931 (2013).

[2] R. B. Meyer, in Molecular Fluids. Les Houches Lectures, 1973, edited by R. Balian and G. Weill (Gordon and Breach, London, 1976), pp. 271-343.

[3] I. Dozov, On the Spontaneous Symmetry Breaking in the Mesophases of Achiral Banana-Shaped Molecules, Europhys. Lett. 56, 247 (2001).

[4] M. Cestari, S. Diez-Berart, D. A. Dunmur, A. Ferrarini, M. R. de la Fuente, D. J. B. Jackson, D. O. Lopez, G. R. Luckhurst, M. A. Perez-Jubindo, R. M. Richardson, J. Salud, B. A. Timimi, and H. Zimmermann, Phase Behavior and Properties of the Liquid-Crystal Dimer 1",7"-bis(4cyanobiphenyl-4'-yl) Heptane: A Twist-Bend Nematic Liquid Crystal, Phys. Rev. E 84, 031704 (2011).

[5] V. Borshch, Y.-K. Kim, J. Xiang, M. Gao, A. Jakli, V. P. Panov, J. K. Vij, C. T. Imrie, M. G. Tamba, G. H. Mehl, and O. D. Lavrentovich, Nematic Twist-Bend Phase with Nanoscale Modulation of Molecular Orientation, Nat. Commun. 4, 2635 (2013).

[6] A. Ferrarini, G. R. Luckhurst, P. L. Nordio, and S. J. Roskilly, Understanding the Unusual Transitional Behaviour of Liquid Crystal Dimers, Chem. Phys. Lett. 214, 409 (1993); Prediction of the Transitional Properties of Liquid Crystal Dimers. A Molecular Field Calculation Based on the Surface Tensor Parametrization, J. Chem. Phys. 100, 1460 (1994).

[7] C. Meyer, G. R. Luckhurst, and I. Dozov, The Temperature Dependence of the Heliconical Tilt Angle in the Twist Bend Phase of the Odd Dimer CB7CB, J. Mater. Chem. C 3, 318 (2015).

[8] A. B. Harris, R. D. Kamien, and T. C. Lubensky, Molecular Chirality and Chiral Parameters, Rev. Mod. Phys. 71, 1745 (1999).

[9] R. B. Meyer, Piezoelectric Effects in Liquid Crystals, Phys. Rev. Lett. 22, 918 (1969).

[10] C. Meyer, G. R. Luckhurst, and I. Dozov, Flexoelectrically Driven Electroclinic Effect in the Twist-Bend Nematic Phase of Achiral Molecules with Bent Shapes, Phys. Rev. Lett. 111, 067801 (2013).

[11] S. M. Shamid, S. Dhakal, and J. V. Selinger, Statistical Mechanics of Bend Flexoelectricity and the Twist-Bend Phase in Bent-Core Liquid Crystals, Phys. Rev. E 87, 052503 (2013).

[12] K. Adlem, M. Copic, G. R. Luckhurst, A. Mertelj, O. Parri, R. M. Richardson, B. D. Snow, B. A. Timimi, R. P. Tuffin, and D. Wilkes, Chemically Induced Twist-Bend Nematic
Liquid Crystals, Liquid Crystal Dimers, and Negative Elastic Constants, Phys. Rev. E 88, 022503 (2013).

[13] T. C. Lubensky, Hydrodynamics of Cholesteric Liquid Crystals, Phys. Rev. A 6, 452 (1972).

[14] L. Radzihovsky and T. C. Lubensky, Nonlinear Smectic Elasticity of Helical State in Cholesteric Liquid Crystals and Helimagnets, Phys. Rev. E 83, 051701 (2011).

[15] P. K. Challa, O. Parri, C. T. Imrie, S. Sprunt, J. T. Gleeson, O. Lavrentovich, and A. Jakli, Twist-Bend Nematic Liquid Crystals in High Magnetic Fields, Phys. Rev. E 89, 060501 (R) (2014).

[16] S. M. Salili, C. Kim, S. Sprunt, J. T. Gleeson, O. Parri, and A. Jakli, Flow Properties of a Twist-Bend Nematic Liquid Crystal, RSC Adv. 4, 57419 (2014).

[17] C. Meyer and I. Dozov, Local Distortion Energy and Coarse-Grained Elasticity of the Twist-Bend Nematic Phase, Soft Matter 12, 574 (2016).

[18] I. Lelidis and G. Barbero, Nematic Phases with Spontaneous Splay-Bend Deformation: Standard Elastic Description, Liq. Cryst. 43, 208 (2016).

[19] E. I. Kats and V. V. Lebedev, Landau Theory for Helical Nematic Phases, JETP Lett. 100, 110 (2014).

[20] E. Virga, Double-Well Elastic Theory for Twist-Bend Nematic Phases, Phys. Rev. E 89, 052502 (2014).

[21] G. Barbero, L. R. Evangelista, M. P. Rosseto, R. S. Zola, and I. Lelidis, Elastic Continuum Theory: Toward Understanding of the Twist-Bend Nematic Phases, Phys. Rev. E 92, 0350501(R) (2015).

[22] C. Greco, G. R. Luckhurst, and A. Ferrarini, Molecular Geometry, Twist-Bend Nematic Phases and Unconventional Elasticity: A Generalized Maier-Saupe Theory, Soft Matter 10, 9318 (2014).

[23] V. P. Panov, M. Nagaraj, J. K. Vij, Y. P. Panarin, A. Kohlmeier, M. G. Tamba, R. A. Lewis, and G. H. Mehl, Spontaneous Periodic Deformations in Nonchiral Planar-Aligned Bimesogens with a Nematic-Nematic Transition and a Negative Elastic Constant, Phys. Rev. Lett. 105, 167801 (2010).

[24] Z. Li and O.D. Lavrentovich, Surface Anchoring and Growth Pattern of the Field-Driven First-Order Transition in a Smectic-A Liquid Crystal, Phys. Rev. Lett. 73, 280 (1994).

[25] A. Jakli and A. Saupe, Electrically Induced Uniform Planar Alignment of $S_{C}^{*}$ Liquid Crystals on Homeotropically Treated Plates, Appl. Phys. Lett. 65, 2777 (1994).

[26] This form is motivated in part by the results of the Monte Carlo simulations reported in Ref. [11]. In principle, for finite $\eta$ in Eq. (1), we could consider an equilibrium component of $\mathbf{p}$ along $\hat{\mathbf{n}}$. If we include such a term $\left(p_{1} \hat{\mathbf{n}}\right)$ in Eq. (3), we find that the additional contribution to the free energy $\left(F_{\mathrm{NTB}}\right)$ is $\left(\frac{1}{2} \mu+\eta\right) p_{1}^{2}+\frac{1}{4} \nu p_{1}^{2}\left(p_{1}^{2}+2 p_{0}^{2}\right)+\frac{1}{2} \kappa q_{0}^{2} p_{1}^{2} \sin ^{2} \beta$. The coefficient of the first term may be written $\frac{1}{2} \mu_{0}\left(T-T_{1}\right)$, where $T_{1}=T_{0}-2 \eta / \mu_{0}$. Thus, to minimize $F_{\mathrm{NTB}}, p_{1}=0$ unless $T<T_{1}$. For large $\eta / \mu_{0}$, as is indicated by our results (see discussion in Sec. V), this range of $T$ will not be experimentally accessible.

[27] The temperature dependence of the hydrodynamic director modes on the nematic side has previously been explored on a different dimer material-see Ref. [12]. Results for this dependence on the mixture studied in the present work are similar to those presented in this reference. 
[28] P.-G. de Gennes and J. Prost, The Physics of Liquid Crystals, 2nd ed. (Oxford, New York, 1993), Chapters 6 and 10.

[29] See Supplemental Material at http://link.aps.org/ supplemental/10.1103/PhysRevX.6.021041 for dynamic visualizations of the fluctuation modes shown in this figure.

[30] Interestingly, a double exponential decay of the DLS correlation function, with a nonhydrodynamic fast component, was observed many years ago in an ordinary rodlike nematic (8CB) [see S. Tripathi, H. Zhong, R. G. Petschek, and C. Rosenblatt, Fast Relaxation Mode in a Thermotropic Uniaxial Nematic Liquid Crystal, Phys. Rev. E 52, 5004 (1995)]. These authors ultimately attributed the fast process (which had a temperature-independent relaxation rate comparable to the rate we observe) to biaxial correlations among molecules and/or to molecular conformational fluctuations with a large biaxial character. In the case of the bent-shaped LC dimers, one can imagine a natural connection between conformational motions and a polar (vector) order parameter that drives the $\mathrm{N}$ to $\mathrm{N}_{\mathrm{TB}}$ transition. Polar correlations in the plane perpendicular to the main director possess a biaxial character but are characterized by a vector rather than second-rank tensor order parameter.

[31] H. Birecki, R. Schaetzing, F. Rondelez, and J. D. Litster, Light-Scattering Study of a Smectic-A Phase near the Smectic-A-Nematic Transition, Phys. Rev. Lett. 36, 1376 (1976).

[32] J. Litster, J. Als-Nielsen, R. Birgeneau, S. Dana, D. Davidov, F. Garcia-Golding, M. Kaplan, C. Safinya, and R. Schaetzing, High Resolution X-Ray and Light Scattering
Studies of Bilayer Smectic-A Compounds, J. Phys. (Paris), Colloq. 40, C3 (1979).

[33] H. von Kanel and J. D. Litster, Light-Scattering Studies on the Single-Layer Smectic p-butoxybenzilidene p-octylaniline, Phys. Rev. A 23, 3251 (1981).

[34] N. A. Clark, Pretransitional Mechanical Effects in a Smectic-A Liquid Crystal, Phys. Rev. A 14, 1551 (1976).

[35] M. R. Fisch, L. B. Sorenson, and P. S. Pershan, Absolute Measurement of the Critical Properties of Second Sound at the Smectic-A-to-Nematic Phase Transition in 8OCB, Phys. Rev. Lett. 47, 43 (1981).

[36] P. Sonntag, D. Collin, and P. Martinoty, Critical Behavior of the de Gennes Elastic Constants near the Nematic-SmecticA Transition of TBBA, Phys. Rev. Lett. 85, 4313 (2000).

[37] Using the values of cone angle $\beta$ and pitch wavenumber $q_{0}$ from Sec. V, assuming $K_{2}$ and the "bare" $K_{3}$ (maximum value in the nematic phase) are in the $\mathrm{pN}$ range (see Ref. [5]), and neglecting the contribution of $\kappa$ (polarization elastic constant), one estimates from Eq. (26) that $B_{\text {eff }}$ should be about 100 times lower in the $\mathrm{N}_{\mathrm{TB}}$ phase than in the smectic-A phase of a typical thermotropic liquid crystal. This estimate agrees with recent experimental results in Ref. [16]; however, this reference does not report on the temperature dependence of $B_{\text {eff }}$.

[38] S. A. Pardaev, S. M. Shamid, M. G. Tamba, C. Welch, G. H. Mehl, J. T. Gleeson, D. W. Allender, J. V. Selinger, B. Ellman, A. Jakli, and S. Sprunt, Second Harmonic Light Scattering Induced by Defects in the Twist-Bend Nematic Phase of Liquid Crystal Dimers, Soft Matter, 12, 4472 (2016), 